Les Cahiers de droit

Les médecins comme travailleurs autonomes au sein du système public de santé au Québec : le passage d'une autonomie professionnelle à une imputabilité sociale Physicians as Self-Employed Workers in Québec's Public Health System : from Professional Autonomy to Social Accountability Los médicos, trabajadores autónomos en el seno del sistema público de salud de Quebec ; el tránsito de una autonomía profesional hacia una imputabilidad social

\author{
Anne-Marie Savard
}

Volume 58, numéro 4, décembre 2017

Le contenu de ce texte est à jour en août 2017.

URI : https://id.erudit.org/iderudit/1042757ar

DOI : https://doi.org/10.7202/1042757ar

Aller au sommaire du numéro

Éditeur(s)

Faculté de droit de l'Université Laval

ISSN

0007-974X (imprimé)

1918-8218 (numérique)

Découvrir la revue

Citer cet article

Savard, A.-M. (2017). Les médecins comme travailleurs autonomes au sein du système public de santé au Québec : le passage d'une autonomie professionnelle à une imputabilité sociale. Les Cahiers de droit, 58(4), 749-787. https://doi.org/10.7202/1042757ar
Résumé de l'article

L'article qui suit porte sur l'évolution historique de la régulation entourant la pratique médicale, qui a permis le passage de l'autonomie professionnelle des médecins à leur imputabilité dans le système de santé, particulièrement en matière d'accès aux soins et aux services. L'auteure démontre d'abord que, bien que la pratique de la médecine ait pris un tournant majeur avec la mise en place d'un système public de santé au début des années 70, les médecins auront néanmoins réussi à conserver une marge de manoeuvre appréciable dans la pratique de leur profession, et ce, au cours des 30 premières années. L'auteure révèle ensuite que la présence de pressions financières de plus en plus importantes, l'émergence d'une nouvelle gestion publique au sein de l'administration publique québécoise et l'introduction d'une philosophie managériale dans le système de santé ont poussé l'État à contraindre toujours plus la pratique médicale, plus particulièrement à partir des années 2000. Cette nouvelle orientation, misant notamment sur la gestion par les résultats, la transparence et la reddition de comptes, a incontestablement rendu possible l'adoption inédite de la Loi édictant la Loi favorisant l'accès aux services de médecine de famille et de médecine spécialisée et modifiant diverses dispositions législatives en matière de procréation assistée (Projet de loi ${ }^{0} 20$ ) ainsi que la conclusion d'ententes entre le ministre de la Santé et des Services sociaux et les fédérations médicales, consacrant désormais une imputabilité des médecins à l'égard du gouvernement, et ultimement de la population, en matière d'accès aux soins et aux services de santé. 


\section{Les médecins comme travailleurs autonomes au sein du système public de santé au Québec: le passage d'une autonomie professionnelle à une imputabilité sociale}

L'article qui suit porte sur l'évolution historique de la régulation entourant la pratique médicale, qui a permis le passage de l'autonomie professionnelle des médecins à leur imputabilité dans le système de santé, particulièrement en matière d'accès aux soins et aux services. L'auteure démontre d'abord que, bien que la pratique de la médecine ait pris un tournant majeur avec la mise en place d'un système public de santé au début des années 70, les médecins auront néanmoins réussi à conserver une marge de manœuvre appréciable dans la pratique de leur profession, et ce, au cours des 30 premières années. L'auteure révèle ensuite que la présence de pressions financières de plus en plus importantes, l'émergence d'une nouvelle gestion publique au sein de l'administration publique québécoise et l'introduction d'une philosophie managériale dans le système de santé ont poussé l'État à contraindre toujours plus la pratique médicale, plus particulièrement à partir des années 2000. Cette nouvelle orientation, misant notamment sur la gestion par les résultats, la transparence et la reddition de comptes, a incontestablement rendu possible l'adoption inédite de la Loi édictant la Loi favorisant l'accès aux services de médecine de famille et de médecine spécialisée et modifiant diverses dispositions législatives en matière de procréation assistée (Projet de loi $\left.n^{o} 20\right)$ ainsi que la conclusion d'ententes entre le ministre de la Santé et des Services sociaux et les fédérations médicales, consacrant

* Professeure, Faculté de droit, Université Laval.

Le contenu de ce texte est à jour en août 2017. 
désormais une imputabilité des médecins à l'égard du gouvernement, et ultimement de la population, en matière d'accès aux soins et aux services de santé.

Physicians as Self-Employed Workers in Québec's Public Health System: from Professional Autonomy to Social Accountability

This article looks at how regulation of the medical profession has evolved over time, moving from professional autonomy for doctors to accountability in the health system, in particular as regards access to care and services. The author shows that, although the practice of medicine underwent a radical shift in the early 1970s when the public health system was established, doctors managed to retain a considerable degree of latitude in the practice of their profession for the first 30 years. Next, she reveals how growing financial pressures, the emergence of New Public Management (NPM) in Québec's public administration, and the introduction of a managerial philosophy within the health system, led the administration to increase the constraints on medical practice, beginning in the 2000s. This new approach, focused in particular on results-based management, transparency and accountability, clearly made possible the passage of the Act to enact the Act to promote access to family medicine and specialized medicine services and to amend various legislative provisions (Bill 20) and the signing of agreements between the Minister of Health and Social Services and the medical federations. It makes doctors accountable to the government, and ultimately to the general public, for access to health care and services.

Los médicos, trabajadores autónomos en el seno del sistema público de salud de Quebec; el tránsito de una autonomía profesional hacia una imputabilidad social

Este artículo trata sobre la evolución histórica de la regulación que circunda la práctica de la medicina, y que ha permitido el paso de la autonomía profesional de los médicos a su imputabilidad en el sistema de 
salud, específicamente en lo que respecta el acceso a la atención médica y servicios. La autora expone inicialmente que si bien la práctica de la medicina alcanzó un gran cambio con el establecimiento de un sistema público de salud a principios de 1970, no obstante, los médicos lograron conservar un considerable margen de maniobra en la práctica de su profesión durante los primeros treinta años. Seguidamente, la autora expone que debido a la presencia de presiones financieras cada vez más importantes, el surgimiento de una nueva gestión pública en el seno de la administración pública quebequense, y la introducción de una filosofía administrativa en el seno del sistema de salud han inducido al Estado a restringir cada vez más el ejercicio de la medicina a partir de los años 2000. Esta nueva orientación, que opta particularmente por la gestión basada en los resultados, la transparencia y la rendición de cuentas, ha logrado indiscutiblemente la adopción sin precedentes del proyecto de Ley nro. 20 Loi édictant la Loi favorisant l'accèes aux services de médicine de famille et de médecine spécialisée et modifiant diverses dispositions législatives en matière de procréation assistée -Ley que promulga la Ley que facilita el acceso a los servicios de medicina familiar y de medicina especializada y que modifica diversas disposiciones legislativas en materia de procreación asistida- así como la suscripción de acuerdos entre el ministro de la Santé et des Services sociaux - Sanidad y Servicios Sociales -y las federaciones médicas, en los que se establece una imputabilidad por parte de los médicos ante el gobierno, y en última instancia, ante la población, en materia de acceso a la atención y servicios de salud.

1 La période 1970-1999: un rapport de force État-médecins relativement équilibré, une autonomie partiellement préservée.

1.1 Des compromis historiques scellant l'autonomie professionnelle des médecins lors de la mise en place de l'assurance maladie

1.2 La restriction des ressources budgétaires forçant un encadrement normatif grandissant de la pratique médicale.

2 La période 2000-2017 : un rapport de force État-médecins davantage déséquilibré, une imputabilité finalement imposée

2.1 L'orientation du système de santé vers une philosophie managériale.

2.2 L'obligation (inédite) de reddition de comptes introduite par la présentation d'un projet de loi 
À peine quelques semaines après les élections québécoises d'avril 2014 ayant porté au pouvoir le Parti libéral, le nouveau ministre de la Santé et des Services sociaux, Gaétan Barrette, affichait ses couleurs. Affirmant que tous les acteurs du réseau de la santé devraient participer aux efforts de redressement des finances publiques, il ajoutait que les médecins ne seraient pas en reste et «ne [pourraient] se soustraire à une obligation de résultat ${ }^{1} \gg$. Dans les faits, le gouvernement a alors entrepris des réformes importantes dans le secteur de la santé, dont celle qui touchait particulièrement les médecins, avec l'adoption le 10 novembre 2015 du Projet de loi $\mathrm{n}^{\mathrm{o}} 20^{2}$, ainsi que la conclusion d'ententes entre le ministre de la Santé et des Services sociaux et les fédérations médicales, par la suite. Ces textes consacraient donc, pour la première fois, un lien d'imputabilité entre les médecins et le gouvernement en matière d'accès aux soins et aux services de santé. Le gouvernement a réussi en effet, de manière inédite, à exiger du corps médical qu'il fournisse désormais des résultats et qu'il lui rende des comptes quant à l'accès de la population aux services de santé. Cependant, comment expliquer que les médecins, qui sont des professionnels et, au surplus, au Québec, des travailleurs autonomes, puissent devenir redevables par rapport à d'autres instances que leurs propres patients? Comment envisager que, jouissant traditionnellement d'une autonomie professionnelle pratiquement sans borne, ils pourraient être aujourd'hui forcés d'améliorer le niveau d'accessibilité aux soins?

Selon une approche historique du système de santé au Québec, nous nous intéressons, dans le présent texte, à la longue évolution ayant permis le passage de l'autonomie professionnelle dont ont traditionnellement disposé les médecins depuis la mise en place du régime public en 1970 jusqu'à l'imposition récente d'un processus d'imputabilité en matière d'accès aux soins et aux services de santé. Dans la première partie, nous passons en revue les 30 premières années d'existence du système public de santé afin de démontrer que, bien qu'ils aient exercé leur profession dans une structure organisée et financée par l'État depuis 1970 et que cela ait entraîné une régulation de plus en plus accrue de leur pratique médicale, les médecins ont néanmoins su préserver une part importante de leur autonomie

1. Marco BÉlAIR-CIRINO, «Santé: Barrette annonce un virage musclé», Le Devoir, 10 mai 2014, [En ligne], [www.ledevoir.com/politique/quebec/407915/la-methode-barrette-pas1-apocalypse-mais-un-changement-de-direction-clair] (13 septembre 2017).

2. Loi édictant la Loi favorisant l'accès aux services de médecine de famille et de médecine spécialisée et modifiant diverses dispositions législatives en matière de procréation assistée, L.Q. 2015, c. 25, édictant la Loi favorisant l'accès aux services de médecine de famille et de médecine spécialisée, RLRQ, c. A-2.2 (pour des raisons de commodité, nous ferons référence à cette loi en employant le numéro du projet de loi dont elle constitue l'aboutissement: «Projet de loi $\left.\mathrm{n}^{\circ} 20 »\right)$. 
professionnelle. Cependant, nous montrons, dans la seconde partie, que la restriction des ressources budgétaires de l'État, amorcée durant les années 80 et ayant finalement induit une philosophie managériale au sein de l'appareil étatique au début des années 2000, ainsi que les hausses importantes de rémunération des médecins auront pavé la voie pour changer la donne jusqu'à leur imposer tout récemment des obligations de rendement et un système de reddition de comptes.

\section{La période 1970-1999: un rapport de force État-médecins relativement équilibré, une autonomie partiellement préservée}

Dans son ouvrage intitulé La profession médicale, le sociologue américain Eliot Freidson définit l'autonomie professionnelle comme «le droit exclusif de décider qui est autorisé à accomplir le travail et comment celui-ci doit l'être ${ }^{3} »$. En d'autres termes, les professionnels sont appelés à déterminer eux-mêmes les exigences universitaires et pratiques à retenir pour autoriser l'exercice de telle profession de même que les standards et les règles de l'art de cette profession. Enfin, ils doivent exercer un contrôle interne de la pratique de leurs membres. Cette facette de l'autonomie professionnelle, que Freidson appelle l'autonomie «technique», est toutefois à distinguer, selon lui, d'une autre facette, l'autonomie «socioéconomique», qui se réfère aux modalités de la pratique professionnelle pouvant interférer avec différentes contraintes sociales, notamment économiques ou éthiques. Ce sera, par exemple, la liberté pour un professionnel de choisir son lieu et son ou ses types de pratique. C'est principalement de la seconde perspective de l'autonomie professionnelle qu'il sera question dans notre article.

À titre de professionnels, les médecins québécois jouissent bien sûr d'une autonomie, consacrée lors de la création de leur premier ordre professionnel, en 1847, soit le Collège des médecins et chirurgiens du BasCanada ${ }^{4}$. Comme nous le verrons dans la première partie de notre article,

3. Eliot Freidson, La profession médicale, Paris, Payot, 1984, p. 81. Au début des années 2000, dans sa thèse consacrée aux transformations des systèmes de soins de santé et aux pratiques d'imputabilité, Carl-Ardy Dubois soutient que l'autonomie professionnelle octroie à un groupe d'individus le droit de définir ses propres standards, les modes d'organisation de son travail et de réguler l'activité de ses membres : Carl-Ardy DuBoIs, Transformation des systèmes de santé et pratiques d'imputabilité, thèse de doctorat, Montréal, Faculté des études supérieures, Université de Montréal, 2002, p. 218. Voir aussi Ezekiel J. EmANuEl et Linda L. EMANuel, «What Is Accountability in Health Care?», Annals of Internal Medicine, vol. 124, n 2, 1996, p. 229, à la page 233.

4. Acte pour incorporer les Membres de la Profession Médicale dans le Bas-Canada, et régler l'étude et la pratique de la Médecine et de la Chirurgie en icelui, Statuts de la Province du Canada, 1847, 10 \& 11 Vict., c. 26. Pour une étude approfondie 
bien que la pratique de la médecine ait pris un tournant majeur avec la mise en place d'un système public de santé au début des années 1970, les médecins auront néanmoins su tirer leur épingle du jeu en s'assurant de conserver une marge de manœuvre appréciable dans la pratique de leur profession (1.1). Cependant, quelques années plus tard, avec les difficultés financières qu'a commencé à subir l'État québécois, de nouvelles politiques publiques et certaines dispositions législatives ont contraint davantage et petit à petit l'action et la pratique des médecins, ce qui a diminué ainsi leur autonomie professionnelle (1.2).

\subsection{Des compromis historiques scellant l'autonomie professionnelle des médecins lors de la mise en place de l'assurance maladie}

Bien que toutes les professions jouissent d'une autonomie professionnelle, force est d'admettre que les médecins sont au surplus investis d'un véritable pouvoir médical dans la société ${ }^{5}$, fruit d'une longue évolution historique de plusieurs siècles au cours desquels, progressivement et difficilement, la médecine a écarté tous les guérisseurs concurrents pour imposer sa domination sur le diagnostic et le traitement des maladies ${ }^{6}$. Au Québec, comme nous l'avons mentionné plus haut, la médecine est devenue officiellement une profession en 1847, et c'est à partir de la fin du XIX ${ }^{\mathrm{e}}$ siècle, à la faveur de conditions épistémologiques et sociales propices au renforcement de ses prérogatives en matière de contrôle des actes médicaux, que cette profession a commencé à s'accaparer - jusqu'à le détenir entièrement - le monopole des soins ${ }^{7}$. Sur ce dernier aspect, l'adoption en 1909 de la Loi

sur la médecine au Québec et l'historique de la création du Collège des médecins et chirurgiens du Bas-Canada, voir Sylvio LEBLOND, «La médecine dans la province de Québec avant 1847 », Les Cahiers des dix, n $^{\circ} 35,1970$, p. 69.

5. Sur ce sujet, voir généralement E. Freidson, préc., note 3. Voir aussi Aude-Claire Fourot, Les représentations du pouvoir médical au Québec, mémoire de maîtrise, Québec, Faculté des études supérieures, Université Laval, 2002. Au Québec, voir: Joseph FACAL, Volonté politique et pouvoir médical. La naissance de l'assurance maladie au Québec et aux États-Unis, Montréal, Les Éditions du Boréal, 2006; Raymond Hudon, Rachel MATHIEU et Élisabeth MARTIN, «Pouvoir médical et interventions législatives au Québec, 2001-2008», Recherches sociographiques, vol. 50, n 2, 2009, p. 255; Raymond Hudon, «Le pouvoir médical au Québec. Tensions autour du statut professionnel des médecins», Recherches sociographiques, vol. 50, n 2, 2009, p. 245 ; Danielle D'Amour, Dominique Tremblay et Michelle ProulX, «Déploiement de nouveaux rôles infirmiers au Québec et pouvoir médical», Recherches sociographiques, vol. 50, nº 2, 2009, p. 301.

6. Nicolas Dodier et Sébastien DARBON, recension de La profession médicale d'Eliot Freidson, Sciences sociales et santé, vol. 3, n 1, 1985, p. 129, à la page 129.

7. Voir Denis Goulet, «Professionnalisation et monopolisation des soins : le Collège des médecins du Québec 1847-1940», Ruptures, revue transdisciplinaire en santé, vol. 10, $\mathrm{n}^{\mathrm{o}} 1,2004$, p. 39. L'auteur présente et analyse la chronologie des évènements entourant 
amendant et refondant la loi relative aux médecins et chirurgiens de la province de Québec ${ }^{8}$ a constitué une étape importante en délimitant de manière plus précise la notion d'exercice de la médecine, ce qui a eu pour effet d'exclure la majorité des concurrents des pratiques diagnostiques et curatives ainsi que de faciliter l'adoption de mesures répressives à leur endroit ${ }^{9}$. Aude-Claire Fourot parle du fruit de cette longue quête d'un monopole comme de l'acquisition d'une légitimité traditionnelle à laquelle s'ajoute une légitimité charismatique, par la dévotion qu'inspiraient la position sociale et le champ d'exercice des médecins. Elle ajoute que, «[e]n étant confronté à des questions de vie et de mort, le médecin était respecté et provoquait une forte emprise émotionnelle chez les personnes qui le rencontraient ${ }^{10}$ ».

Ainsi, juste avant que l'État décide d'intervenir de manière draconienne dans le champ de la santé en instaurant des systèmes d'assurance hospitalisation et maladie ${ }^{11}$ et sans nier le travail fondamental des communautés religieuses, sur le plan professionnel, le médecin dominait largement la prestation des soins de santé. Sur le plan du type de pratique médicale, Joseph Facal souligne que, «jusqu'aux années 1960, le seul véritable archétype du médecin pratiquant sera celui de l'entrepreneur indépendant, payé à l'acte, et sans autres contraintes que celles qu'il s'impose lui-même ou celles établies par ses pairs ${ }^{12}{ }$. C'est l'époque de la médecine libérale à l'état pur, se caractérisant par une liberté quasi absolue en matière de prestation, de contrôle et de régulation des soins médicaux. En contrepartie du pouvoir qui leur était accordé de contrôler ces aspects clés ainsi que

la professionnalisation et la monopolisation des soins par les médecins québécois, en quatre étapes différentes: 1) la phase d'émergence du corporatisme médical (17881847); 2) la phase d'implantation de la profession médicale (1847-1876); 3) la phase de stabilisation de la profession (1876-1909); et 4) la phase d'expansionnisme conquérant de la profession (1909-1940).

8. Loi amendant et refondant la loi relative aux médecins et chirurgiens de la province de Québec, S.Q. 1909, c. 55.

9. D. Goulet, préc., note 7, p. 43. Cet auteur explique également que la Loi amendant et refondant la loi relative aux médecins et chirurgiens de la province de Québec, S.Q. 1909, a eu pour effet d'augmenter les pouvoirs du Collège des médecins relativement à la régie interne de la profession et au contrôle de l'offre de soins.

10. A.-C. Fourot, préc., note 5, p. 77.

11. Il faut toutefois souligner qu'avant 1960 l'État québécois est intervenu à quelques reprises dans le domaine sanitaire, notamment en 1921, par l'adoption de la $L o i$ établissant le service de l'assistance publique de Québec, S.Q. 1921, c. 79, qui prévoit le partage des coûts d'hospitalisation et d'internement des malades, des indigents et des orphelins entre les institutions, les municipalités et le gouvernement provincial.

12. J. FACAL, préc., note 5, p. 33. 
leurs conditions de travail, on s'attendait toutefois que les médecins maintiennent des normes élevées de compétence et de responsabilité morale ${ }^{13}$.

Le 9 novembre 1966 est instituée, par le gouvernement Jean Lesage, la Commission d'enquête sur la santé et le bien-être social, aussi appelée «commission Castonguay-Nepveu ${ }^{14} »$. Dans le contexte de son mandat général, qui consistait à examiner le bien-fondé de la mise en place d'un système public d'assurance maladie au Québec, la Commission s'est aussi penchée sur l'exercice de la médecine libérale, comme nous le verrons. Soulignons d'abord que, dans son rapport rédigé en collaboration avec Gérard Nepveu, Claude Castonguay recommande principalement la mise en œuvre d'un régime d'assurance maladie complet et universel pour tous les résidents du Québec. D'une part, il faut se rappeler que, déjà, la $L o i$ de l'assurance-hospitalisation ${ }^{15}$ et la Loi de l'assistance médicale $e^{16}$ étaient respectivement entrées en vigueur en 1961 et en 1966, la première pour assurer la couverture publique des soins hospitaliers pour tous et la seconde pour garantir la couverture des soins médicaux aux assistés sociaux. D'autre part et plus globalement, le Québec était alors en pleine Révolution tranquille, période de «modernisation accélérée des institutions québécoises, animée à la fois par une rationalité technocratique et une soif de justice sociale ${ }^{17} \gg$. Dans ce contexte, l'introduction de l'assurance maladie semblait aller de soi et surtout s'opposait à la pratique de la médecine libérale, telle qu'elle s'exerçait jusqu'alors dans la province.

En effet, la réforme proposée par Castonguay cherchait à faire en sorte que l'État puisse se donner une véritable politique de la santé, dans un nouveau système où il ne s'agirait plus simplement de fournir des soins, mais bien de miser sur la prévention, les soins continus et la réhabilitation $^{18}$. Dans ce contexte, les réformateurs ont également inséré dans leur rapport une mise en accusation de la médecine traditionnelle, en prônant le passage d'une emprise corporative (de la part des médecins et des intérêts privés) sur le domaine de la maladie à une emprise étatique sur le domaine

13. William M. Sullivan, «Medicine under Threat: Professionalism and Professional Identity», Canadian Medical Association Journal, vol. 162, n ${ }^{\circ}$ 5, 2000, p. 673, à la page 673 .

14. Plus particulièrement, le mandat de la Commission était de produire un rapport sur la situation des soins de santé et des services sociaux, qui, à l'époque, étaient sous le contrôle du clergé. Voir Gouvernement du QUÉBeC, Rapport de la Commission d'enquête sur la santé et le bien-être social, Québec, Gouvernement du Québec, 1967, 7 vol. (ci-après «rapport Castonguay-Nepveu»).

15. Loi de l'assurance-hospitalisation, S.R.Q. 1964, c. 163.

16. Loi de l'assistance médicale, S.Q. 1966, c. 11.

17. J. FACAL, préc., note 5, p. 36.

18. Id., p. 122 . 
de la santé ${ }^{19}$. Louis Demers résume fort bien le profond malaise, voire l'«anachronisme dysfonctionnel», que représentait pour la commission Castonguay la médecine de type libéral:

L'autonomie dont disposent les médecins, notamment dans leur pratique hospitalière et leur mode de rémunération, contribue à l'inefficacité du secteur de la santé. L'autodétermination de la profession médicale est à la source de nombreuses lacunes dont le déséquilibre entre le nombre de médecins spécialistes et d'omnipraticiens, la mauvaise répartition des médecins sur le territoire québécois, la consommation excessive de services hospitaliers (l'hospitalocentrisme), l'accent mis sur les services curatifs au détriment de la prévention et de la santé publique, le cloisonnement entre champs de responsabilité professionnels et la duplication des services entre les établissements ${ }^{20}$.

Bien que ces importantes critiques aient pu laisser présager des changements draconiens concernant la pratique médicale, la forte résistance des médecins et plusieurs facteurs sociopolitiques se conjugueront et forceront le gouvernement à faire d'importantes concessions, ce qui laissera au corps médical une autonomie professionnelle importante, qui marqueront le cours de l'histoire du système de santé québécois. Pour bien saisir ces concessions, il est essentiel de poser les grandes lignes des principaux points d'achoppement existant entre les fédérations médicales $^{21}$ et le gouvernement. D'abord, si le regroupement des médecins omnipraticiens a accueilli plutôt favorablement les recommandations du rapport Castonguay, il a tout de même émis quelques réserves, surtout liées à des craintes que leur statut d'infériorité relativement aux médecins spécialistes ne se cristallise avec l'instauration du régime public ${ }^{22}$. Quant

19. Frédéric Lesemann, Du pain et des services. La réforme de la santé et des services sociaux au Québec, Laval, Éditions coopératives Albert Saint-Martin, 1981, p. 40.

20. Louis Demers, «La profession médicale», dans Vincent Lemieux et autres (dir.), Le système de santé au Québec. Organisations, acteurs et enjeux, Sainte-Foy, Les Presses de l'Université Laval, 1994, p. 213, à la page 218.

21. La Fédération des médecins omnipraticiens du Québec (FMOQ) a été créée en 1963; la Fédération des médecins spécialistes du Québec (FMSQ), en 1965. Ces deux organismes avaient (et ont toujours) pour mission de représenter les intérêts de leurs membres et de négocier avec l'État, particulièrement dans une période de profonds bouleversements. Cela a été la naissance du syndicalisme médical au Québec. Les deux fédérations sont d'ailleurs reconnues, depuis le 17 mars 1966 lors de la signature de la première entente relative à la Loi de l'assistance médicale, préc., note 16, par l'État comme les deux seuls organismes représentatifs des médecins au Québec aux fins des négociations (nous reviendrons sur ce point).

22. Étant donné qu'à l'époque les postes clés du Collège des médecins étaient détenus par des spécialistes, et pensant que les négociations sur les modalités de participation de la profession au sein du nouveau régime public seraient menées par le Collège, les omnipraticiens craignaient que l'issue ne leur soit défavorable (J. FACAL, préc., note 5, p. 55). Ainsi, les revendications de la FMOQ se résumaient ainsi : «1) que la FMOQ soit 
au regroupement des médecins spécialistes, il exigeait de l'État les trois conditions suivantes pour accepter le régime universel:

1) la reconnaissance du droit de pratique en dehors du régime et la possibilité pour le médecin de négocier lui-même ses honoraires;

2) l'assurance que le seul organisme légitime pouvant contrôler tous les aspects de la pratique professionnelle serait le Collège des médecins du Québec;

3) la négociation obligatoire d'une grille de tarification pour chaque acte médical à des taux semblables à ceux de l'Ontario ${ }^{23}$.

Enfin, il va sans dire que les deux fédérations médicales étaient totalement opposées à tout changement du statut du médecin: elles tenaient à ce que ce dernier demeure un travailleur autonome rémunéré à l'acte, même au sein du nouveau système de santé.

Dans un premier temps, le gouvernement québécois a tenté un compromis $^{24}$ en établissant la possibilité pour un médecin de pratiquer en dehors du régime, aussi appelé «principe du droit de retrait» (opting $o u t$ ), mais seulement à la condition qu'un maximum de 3 p. 100 des effectifs médicaux de chaque spécialité - et de chaque région-puisse se désengager du régime. Cette clause a été jugée inacceptable par la Fédération des médecins spécialistes du Québec (FMSQ), laquelle arguait que le désengagement était un «droit fondamental» et qu'ainsi aucune condition ne devrait le restreindre. Par la suite, malgré le retrait par le gouvernement des conditions entourant le désengagement des médecins et bien que la $L o i$ sur l'assurance maladie ${ }^{25}$ ait été tout de même adoptée en juillet 1970, la FMSQ a maintenu son opposition au régime universel et a poursuivi sa campagne de relations publiques, notamment en dénonçant les «libertés fondamentales menacées ${ }^{26}$ » et «l'intrusion de l'État dans la relation thérapeutique $^{27} \gg$. L'opposition des médecins spécialistes au régime d'assurance

reconnue comme seule habilitée à représenter les omnipraticiens;2) que chaque acte médical ait un tarif unique, qu'il soit posé par un omnipraticien ou un spécialiste, et où que ce soit sur le territoire du Québec; et 3) que les omnipraticiens aient davantage accès aux hôpitaux d'enseignement, chasse gardée jusque-là des spécialistes » (J. FACAL, préc., note 5, p. 56).

23. Id., p. 41 .

24. Sur ces aspects, voir Vanessa RoY, Les obstacles juridico-politiques à la collaboration interprofessionnelle en santé et la nécessité de revoir certains modes de gouvernance prévus dans la LSSS, mémoire de maîtrise, Sherbrooke, Faculté de droit, Université de Sherbrooke, 2017.

25. Loi sur l'assurance maladie, L.Q. 1970, c. 37.

26. J. FACAL, préc., note 5, p. 49.

27. Id. 
maladie a par ailleurs conduit à une grève de 17 jours en octobre 1970. Le dénouement de l'histoire aura lieu à cause des tragiques évènements d'octobre 1970 (la crise d'Octobre), qui ont relégué au second plan l'enjeu des négociations entre les médecins et le gouvernement, alors que les médecins spécialistes ont été contraints d'intégrer le système de santé public, en l'absence momentanée de soutien de l'opinion publique à leur égard ${ }^{28}$. La Loi sur l'assurance maladie, qui a établi la gratuité des soins médicaux, est ainsi entrée en vigueur le $1^{\text {er }}$ novembre 1970, laissant toutefois intacts le statut des médecins et leur mode de rémunération.

Une fois les programmes assurantiels (hospitalisation et maladie) mis en place par l'État, il était désormais impératif d'instaurer un cadre normatif pour régir la structure du réseau de la santé et des services sociaux, ce qui a été le cas avec l'entrée en vigueur de la Loi sur les services de santé et les services sociaux (LSSS) ${ }^{29}$, en 1971. Là encore, de nombreuses concessions ont été faites en faveur des médecins exerçant en milieu hospitalier, qui refusaient notamment de devenir subordonnés à une direction administrative, soit celle des services professionnels (DSP). Ainsi, le projet de loi a été modifié, notamment afin qu'il soit établi que les gestionnaires gèrent en interférant le moins possible dans la pratique médicale. Comme Louis Demers le souligne avec justesse, «l'écart entre les mesures contenues dans le rapport de la commission et la version finale du projet de loi 65 [la LSSS] ainsi qu'entre la réforme réelle et celle qu'entrevoyait le législateur témoigne du pouvoir de la profession médicale ${ }^{30}$ ».

En somme, bien que l'entrée en scène d'un tiers agent payeur, l'État, en 1970, ait profondément bouleversé les fondements de la prestation des soins médicaux, il ressort néanmoins que l'autonomie professionnelle des médecins, dont ils disposaient auparavant, n'a pratiquement pas été touchée. En effet, étant donné les importantes concessions faites aux médecins par l'État, c'est-à-dire principalement la préservation de leur statut de travailleurs autonomes et du mode de rémunération à l'acte, tout en les assurant d'une rémunération régulière ${ }^{31}$, ainsi que la possibilité de se désengager du système, les médecins auraient davantage bénéficié que souffert de la

28. $I d$.

29. Loi sur les services de santé et les services sociaux, RLRQ, c. S-4.2 (ci-après «LSSS »).

30. L. Demers, préc., note 20, p. 218.

31. Guillaume HÉBERT, «La rémunération des médecins québécois », Institut de recherche et d'informations socioéconomiques (IRIS), 2016, p. 1, [En ligne], [iris-recherche. s3.amazonaws.com/uploads/publication/file/Re_mune_ration_des_me_decins_WEB. pdf] (15 septembre 2017); J. FACAL, préc., note 5, p. 174; L. Demers, préc., note 20, p. 220. 
prise en charge publique du secteur de la santé ${ }^{32}$. De plus, comme nous le verrons, ces bases, mises en place sur fond d'intenses négociations entre les fédérations médicales et le gouvernement, qui ont cristallisé du même coup des valeurs et des principes importants de part et d'autre, seront difficiles à remettre en question par la suite.

Enfin, un dernier élément appuyant la thèse selon laquelle l'autonomie des médecins a été préservée lors de la période contemporaine de la mise en place du système public de santé mérite d'être souligné. Il est permis d'avancer que l'entrée en vigueur en 1973 du Code des professions ${ }^{33}$, cette loi-cadre du système professionnel québécois, qui réglemente la pratique des professions et précise les conditions d'exercice professionnel, a milité en faveur de cette autonomie professionnelle. En effet, en plaçant les professionnels dans une logique de déférence à l'expertise en échange d'une reddition de comptes de leurs compétences à l'égard de leurs pairs hiérarchiques au sein des ordres professionnels ${ }^{34}$, l'État leur garantissait du même coup la liberté d'exercer leur profession de manière autonome. Quant aux médecins en particulier, tout en leur fournissant les ressources matérielles (du moins pour ceux qui exerçaient dans les établissements de santé) et en les rémunérant, l'État attendait d'eux qu'ils soignent leurs patients et travaillent à améliorer la santé de la population, et qu'ils le fassent avec professionnalisme. Ainsi, au début du système, outre l'encadrement professionnel, peu de régulation existait pour orienter la pratique médicale ${ }^{35}$ dans le but de répondre à des exigences d'efficacité ou d'efficience dans le système, ce qui laissait une large part d'autonomie professionnelle aux médecins. Toutefois, la situation ne durera pas longtemps, comme nous le verrons ci-dessous.

\subsection{La restriction des ressources budgétaires forçant un encadrement normatif grandissant de la pratique médicale}

Quelques années après la mise en place du système public de santé, l'État commence à s'inquiéter du taux de croissance des dépenses de sa mission «santé». En fait, plus globalement, ce sont les contrecoups de l'expansionnisme effréné de l'État québécois dans tous les secteurs économiques et sociaux au cours des dernières années que subit alors

\footnotetext{
32. L. DEMERS, préc., note 20.

33. Code des professions, L.Q. 1973, c. 43.

34. C.-A. DuboIs, préc., note 3 , p. 87 et 88.

35. Association MÉdicAle DU QuÉBEC (AMQ), Le rôle de la profession médicale dans la gouvernance du système de santé québécois, Montréal, 2015, p. 10, [En ligne], [www.amq.ca/images/Prises_de_position/Rapport_Gouvernance_m\%C3\%A9dicale_ FINAL2.pdf] (15 septembre 2017).
} 
le gouvernement. Les dépenses budgétaires de l'État ont triplé de 1961 à $1984^{36}$. Comme le souligne Louis Demers, «[1]'année 1975 marque symboliquement la fin des "Trente Glorieuses" années de croissance économique soutenue qui ont suivi la fin de la Seconde Guerre mondiale ${ }^{37} \gg$. Le gouvernement n'a d'autre choix que de freiner l'augmentation de ses dépenses, particulièrement en matière de santé. Ainsi, le rationnement des ressources commence véritablement en 1976, à l'occasion de la première série de restrictions budgétaires (diminution du nombre de lits hospitaliers destinés aux soins de courte durée, restriction sur les achats d'équipements, etc. $)^{38}$. Évidemment, les médecins ne pourront être épargnés, puisqu'ils constituent la ressource humaine la plus onéreuse du système et que, étant majoritairement rémunérés à l'acte, ils influent énormément sur l'offre et sur les dépenses. En 1976, le gouvernement signe donc une entente ${ }^{39}$ sur les conditions de rémunération avec les fédérations médicales, entente qui est toujours en vigueur aujourd'hui et qui assujettit la masse de la rémunération des médecins à des objectifs tarifaires négociés et, pour les omnipraticiens, à un plafond de revenus trimestriel ${ }^{40}$.

36. Carl-Ardy Dubors et Jean-Louis Denis, De la Commission Castonguay-Nepveu à la Commission Clair. Le façonnement socio-historique de l'imputabilité, 2001, p. 11, [En ligne], [www.getoss.enap.ca/GETOSS/Publications/Lists/Publications/ Attachments/189/De \%20la \%20commission \%20castonguay-Nepveu \%20\%C3\%A0\%20 la \%20commission \%20Clair.pdf] (15 septembre 2017).

37. L. Demers, préc., note 20, p. 221. Dans le même sens, voir C.-A. Dubois, préc., note 3, p. 5 .

38. L. Demers, préc., note 20.

39. RÉGIE DE L'ASSURANCE MALAdIE du QuÉBEC (RAMQ), Brochure $n^{\circ} 1$-Omnipraticiens. Entente relative à l'assurance maladie et à l'assurance hospitalisation entre le ministre de la Santé et des Services sociaux et la Fédération des médecins omnipraticiens du Québec, [En ligne], [www.ramq.gouv.qc.ca/SiteCollectionDocuments/professionnels/ manuels/104-brochure-1-omnipraticiens/004_entente_omni.pdf] (15 septembre 2017); RÉGIE DE L'ASSURANCE MALADIE DU QUÉBEC (RAMQ), «Accord-cadre entre le Ministre de la santé et des services sociaux et la Fédération des médecins spécialistes du Québec aux fins de l'application de la loi sur l'assurance maladie», dans Manuel des spécialistes brochure $n^{\circ} 1$ ( $n^{\circ}$ 154), 2007, [En ligne], [www.ramq.gouv.qc.ca/SiteCollectionDocuments/ professionnels/manuels/154-brochure-1-specialistes/000_complet_acc_cadre_spec. pdf] (15 septembre 2017). L'article 19 de la Loi sur l'assurance maladie, RLRQ, c. A-29, constitue l'assise juridique des ententes entre le ministre de la Santé et des Services sociaux et les fédérations médicales, en ce qu'il permet au ministre, avec l'approbation du Conseil du trésor, de conclure avec les organismes représentatifs de toute catégorie de professionnels de la santé toute entente pour l'application de cette loi.

40. L. DEMERS, préc., note 20, p. 222. Cette entente est alors inédite et se trouve aujourd'hui la cible de nombreuses critiques, puisque c'est lors des négociations relatives à l'ampleur de l'enveloppe budgétaire globale de rémunération entre les fédérations médicales et le ministre de la Santé et des Services sociaux que se décide du même coup le panier de 
L'aspect qui nous intéresse ici est surtout de voir comment cette période de restriction budgétaire et de rationalisation de l'offre de soins a amené le gouvernement à repenser l'organisation du travail des médecins et surtout à vouloir intervenir de plus en plus dans la pratique médicale, dans une perspective d'efficience du système, ce qui diminue du même coup l'autonomie professionnelle des médecins. Sans en faire une revue complètement exhaustive, nous verrons cependant les principaux changements législatifs attestant ce phénomène. En 1981, le parlement adopte une loi ${ }^{41}$ ayant pour objectif de "responsabiliser" les médecins quant aux conséquences budgétaires de leurs décisions ${ }^{42} »$. S'appliquant en milieu hospitalier, cette loi devait renforcer l'autorité du directeur des services professionnels sur les chefs de département clinique (tous les deux devant être des médecins) et l'autorité de ceux-ci sur leurs collègues médecins, concernant la gestion et l'utilisation des ressources de leur département. La subordination à une direction administrative et l'exercice d'une autorité hiérarchique sur leurs collègues, auxquels s'étaient d'ailleurs expressément opposés les médecins lors de l'adoption de la LSSS en 1971, allaient «directement à l'encontre des principes d'autonomie de pratique et de responsabilité individuelle du médecin à l'égard de ses patients qui sont au cœur du système de valeurs médical ${ }^{43}{ }$. Par ailleurs, à partir de 1982, dans le but d'améliorer la répartition géographique des médecins sur le territoire québécois et particulièrement afin d'amener un certain nombre d'entre eux à s'installer et à pratiquer en région, le ministère des Affaires sociales introduit une série de mesures incitatives à caractère économique ${ }^{44}$. Toutefois, ces mesures ne comblant pas les attentes, le Parlement du Québec adopte une loi en $1987^{45}$ afin de restreindre la liberté d'établissement des médecins spécialistes, toujours dans le but d'améliorer leur répartition géographique, selon laquelle le ministère de la Santé déterminera, pour chaque région et pour chaque spécialité médicale, le nombre de médecins ayant le droit d'y pratiquer $^{46}$.

services de santé. À ce sujet, voir notamment Mélanie Bourassa Forcier et autres, L'encadrement juridique du panier de services de santé et de services sociaux au Québec, Montréal, Éditions Yvon Blais, 2016.

41. Loi modifiant diverses dispositions législatives dans le domaine de la santé et des services sociaux, L.Q. 1981, c. 22.

42. L. Demers, préc., note 20, p. 231.

43. Id.

44. Id., p. 225.

45. Loi modifiant la Loi sur les services de santé et les services sociaux, L.Q. 1987, c. 104.

46. C'est la première version de ce que l'on appelle les «plans régionaux d'effectifs médicaux (PREM)» qui prendront plus d'ampleur lors de l'adoption de la LSSS de 1991, comme nous le verrons. 
Par la suite, ce sera principalement l'adoption de la seconde version de la $\operatorname{LSSS}^{47}$ qui entraînera des restrictions à la pratique médicale, diminuant ainsi une partie de leur autonomie professionnelle. Cependant, comme nous le verrons, le pouvoir médical, encore bien présent à l'époque, a limité la marge de manœuvre du Parlement qui aurait souhaité contraindre davantage les médecins, particulièrement quant aux lieux et aux types de pratique; mais d'abord, quel est le contexte ayant mené à l'adoption de la seconde version de la LSSS ? Les lacunes et les problèmes mentionnés plus haut, particulièrement liés à la restriction des ressources financières, et le fait que, selon plusieurs acteurs du réseau, le moment était venu de faire le bilan des premières années d'existence du système public de santé, ont poussé le ministre de la Santé et des Services sociaux de l'époque, Guy Chevrette, à créer la Commission d'enquête sur les services de santé et les services sociaux ${ }^{48}$, en 1985 , connue sous le nom de «commission Rochon $^{49}$ ». Deux ans plus tard, le rapport de la Commission ${ }^{50}$, qui comporte plusieurs propositions de changements, comme l'implantation d'une réforme axée sur le citoyen, la mise en œuvre d'une politique de la santé et du bien-être ainsi qu'une meilleure coordination et une régionalisation accrue des services, est déposé publiquement. Il faudra toutefois attendre encore quatre ans pour que la seconde version de la LSSS soit finalement adoptée $\mathrm{e}^{51}$.

$\mathrm{Au}$ cours de la Commission parlementaire sur l'avant-projet de cette loi se sont dessinées plusieurs orientations clés de la réforme concernant les médecins. Plus particulièrement, c'est à ce moment-là qu'un constat selon lequel il était désormais impératif de toucher au pouvoir et au statut des médecins a été fait ${ }^{52}$. Le Parlement trouvait en effet essentiel de contrôler plus strictement la répartition géographique et les types de pratique des médecins. Au sujet de ce dernier aspect plus précisément, on

47. Loi sur les services de santé et les services sociaux et modifiant diverses dispositions législatives, L.Q. 1991, c. 42.

48. Cette commission a été créée par le Décret 1156-85 relatif à la Commission d'enquête sur la santé et les services sociaux connexes, (1985) 30 G.O. II, 3571.

49. Cette commission était présidée par Jean Rochon, médecin spécialisé en santé publique.

50. COMMISSION D'ENQUÊTE SUR LES SERVICES DE SANTÉ ET LES SERVICES SOCIAUX, Rapport de la Commission d'enquête sur les services de santé et les services sociaux, Québec, Les Publications du Québec, 1988 (ci-après «commission Rochon»).

51. Loi sur les services de santé et les services sociaux et modifiant diverses dispositions législatives, préc., note 47. Pour une étude détaillée du contexte menant à l'adoption de cette loi et qui en trace le cheminement parlementaire complet, voir Michelle GIROUX, Guy Rocher et Andrée LAJOIE, «Droit de la santé. L'émergence de la Loi sur les services de santé et les services sociaux de 1991: une chronologie des événements", (1999) 33 R.J.T. 659.

52. Id., 679 . 
voulait éviter que les omnipraticiens surinvestissent la pratique privée en cabinet au détriment de la pratique en établissement, surtout en salle d'urgence. Évidemment, le corps médical s'est montré très réticent devant ces nouvelles orientations : c'est pour cette raison que, lors de l'étude détaillée du projet de loi en commission parlementaire au printemps et à l'été 1991, de vives négociations ont eu lieu entre le gouvernement et les médecins. Le litige portait sur trois aspects névralgiques pour les médecins, c'est-à-dire leur liberté d'installation, leur autonomie professionnelle et la participation aux décisions les concernant ${ }^{53}$. En marge des négociations, la profession médicale a organisé des manifestations publiques, des ralentissements de travail et des campagnes de sensibilisation et de publicité auprès de la population.

Malgré tout, concernant les types de pratique, le nouveau concept des activités médicales particulières (AMP) a été introduit dans la seconde version de la LSSS en 1991. Les AMP avaient pour objectif d'orienter la pratique médicale vers les secteurs de pratique jugés prioritaires par l'État (l'urgence étant le plus prioritaire), afin de s'assurer que tous les médecins omnipraticiens ne concentrent pas leur pratique en cabinet ${ }^{54}$. De plus,

53. Id., 686 .

54. Alors qu'initialement elles n'étaient applicables qu'aux médecins omnipraticiens titulaires d'un permis de pratique de la Corporation professionnelle des médecins depuis moins de dix ans, les AMP sont, depuis 2002, applicables à tous les omnipraticiens ainsi qu'aux spécialistes n'ayant pas de privilèges dans un établissement qui exploite un centre hospitalier (voir la Loi modifiant la Loi sur les services de santé et les services sociaux concernant les activités médicales, la répartition et l'engagement des médecins, L.Q. 2002, c. 66), le nombre d'heures obligatoires par semaine variant en fonction du nombre d'années de pratique. Notons que l'encadrement législatif des AMP se trouve, depuis 1991, aux articles 360 à 366 de la LSSS, mais les modalités en sont prévues dans des ententes entre le ministre de la Santé et des Services sociaux et les fédérations médicales, les dernières ententes étant entrées en vigueur à la suite de l'adoption du Projet de loi no 20, en 2015. À titre d'illustration, pour les omnipraticiens, les AMP sont aujourd'hui classifiées en trois blocs, lesquels correspondent à des niveaux décroissants de priorité. Le premier bloc constitue la prestation de services médicaux dans un service d'urgence. Le deuxième bloc comprend:

- la prestation, en première ligne, de services médicaux d'inscription et de suivi de clientèle;

- la prestation de soins aux usagers admis en soins de courte durée d'un établissement exploitant un centre hospitalier;

- la prestation, dans le service d'obstétrique d'un centre exploité par un établissement, de services médicaux en obstétrique;

- la prestation de services médicaux, impliquant une garde en disponibilité, dans un centre hospitalier de soins de longue durée (CHSLD), un centre de réadaptation ou dans le contexte d'un programme de soutien à domicile d'un centre local de services communautaires (CLSC). 
concernant les lieux de pratique, les plans régionaux d'effectifs médicaux (PREM), que nous avons mentionnés ci-dessus, ont été étendus aux omnipraticiens. L'objectif de ceux-ci était (et est toujours) de répartir les effectifs médicaux sur le territoire québécois de la manière la plus équitable ${ }^{55}$. La citation suivante d'Aude-Claire Fourot illustre bien, selon nous, ce que l'introduction de ces mesures représentait alors pour les médecins:

\begin{abstract}
Ainsi, nous pouvons considérer que le véritable enjeu de ces séries de mesures est la définition même de l'exercice libéral de la médecine. La médecine libérale confère un statut au médecin qui devrait lui permettre de choisir ses patients et de les référer quand il juge que cela n'est pas de son ressort. L'objectif qui est mis en valeur par la profession est l'autocontrôle du praticien [...] Or, ce modèle de la médecine libérale semble remis en question à la fois pour le patient et pour le médecin ${ }^{56}$.
\end{abstract}

S'il est vrai que l'introduction de ces dispositions législatives a contraint davantage la pratique médicale et a, du même coup, diminué l'autonomie professionnelle des médecins, il importe de préciser que ces derniers ont réussi à freiner significativement les ardeurs du gouvernement et du Parlement. En effet, bien qu'ils n'aient pas obtenu le retrait pur et simple du projet de loi, comme ils le souhaitaient ${ }^{57}$, les médecins ont néanmoins réussi à s'assurer de conserver un droit de regard et de parole non négligeable sur l'application de ces nouvelles mesures et plus, globalement, sur les orientations les concernant. En effet, juste avant l'adoption de la LSSS, à la fin du mois d'août 1991, le gouvernement et les médecins sont parvenus à une entente sur les quatre éléments suivants ${ }^{58}$ :

1) la création du Conseil médical du Québec ${ }^{59}$, composé majoritairement de médecins et ayant «pour fonction de conseiller le ministre sur toute question relative aux services médicaux, en tenant compte des besoins de la population, de l'évolution des coûts des services médicaux et de la capacité de payer de la population»;

Enfin, le troisième bloc comprend toute autre activité jugée prioritaire par le Département régional de médecine générale (DRMG).

55. À noter que si les AMP et les PREM sont prévus par la loi, des ententes entre le ministre de la Santé et des Services sociaux et les fédérations médicales en déterminent les modalités.

56. A.-C. Fourot, préc., note 5, p. 95.

57. M. Giroux, G. Rocher et A. LaJoie, préc., note 51, 687.

58. Id., 689 .

59. Le Conseil a été créé par la Loi sur le Conseil médical du Québec, L.R.Q., c. C-59.0001, adoptée en 1991. Voir particulièrement les articles 16 à 22 pour une description complète des pouvoirs de ce conseil. Notons qu'en 2005 le Commissaire à la santé et au bien-être a été substitué au Conseil médical du Québec et au Conseil de la santé et du bien-être. Voir la Loi sur le Commissaire à la santé et au bien-être, RLRQ, c. C-32.1.1. 
2) la participation des fédérations médicales aux décisions concernant la répartition des médecins sur le territoire;

3) le maintien des conseils des médecins, dentistes et pharmaciens (CMDP) au sein des établissements; et, dans une moindre mesure,

4) l'agrément des cabinets privés et la répartition géographique des médecins.

Ces derniers se sont particulièrement réjouis des deux premiers éléments et «ont salué comme une victoire historique de la profession l'abandon de l'approche hiérarchique du premier projet de loi 120_qui, selon eux, assujettissait les médecins à l'arbitraire des ministres et des administrateurs ${ }^{60} \gg$.

En définitive, au cours des 30 premières années suivant l'introduction du régime public de santé, force est de constater une intervention des pouvoirs publics de plus en plus insistante concernant la pratique médicale, ce qui a évidemment entraîné une diminution, du moins dans une certaine mesure, de l'autonomie professionnelle des médecins ${ }^{61}$. En effet, pour reprendre les distinctions de Freidson, si l'État laisse à la profession médicale son autonomie «technique» ou clinique, il contraint désormais davantage son autonomie «socioéconomique», c'est-à-dire les modalités de sa pratique, et ce, pour tenter de mieux contrôler et rationner les dépenses publiques ainsi que pour répartir le mieux possible les effectifs médicaux en fonction des secteurs et des lieux géographiques requérant le plus de services. Malgré tout, l'analyse du processus législatif de la LSSS de 1991 nous force à reconnaître que le pouvoir médical était encore très présent au sein de la société québécoise au tournant des années 90. Michelle Giroux, Guy Rocher et Andrée Lajoie concluent d'ailleurs ainsi sur la seconde réforme importante du réseau de la santé: «Cette forme "corporatiste" d'action législative fut évidemment l'effet boomerang d'un projet de réforme entrepris par l'État pour réduire le pouvoir d'un corps médical, qui demeure cependant toujours capable de se gagner l'opinion publique ${ }^{62}$.»

Finalement, ce pouvoir médical arrivait à tenir en équilibre les rapports de force entre le gouvernement et les médecins et assurait à ces derniers de ne pas voir leur pratique médicale entièrement contrôlée par l'État. Comme nous le verrons dans la seconde partie de notre article,

60. L. DEMERS, préc., note 20, p. 233.

61. A.-C. Fourot, préc., note 5, p. 13. Dans le même sens, voir aussi Richard L. Cruess et Sylvia R. Cruess, «Expectations and Obligations. Professionalism and Medicine's Social Contract with Society», Perspectives in Biology and Medicine, vol. 51, $\mathrm{n}^{\circ} 4$, 2008 , p. 579 , à la page 589.

62. M. Giroux, G. Rocher et A. Lajoie, préc., note 51, 692. 
plusieurs phénomènes se conjugueront au cours des années 2000 et feront en sorte de donner à l'État la marge de manœuvre nécessaire non seulement pour diminuer encore plus l'autonomie professionnelle des médecins, mais également pour leur demander de lui rendre des comptes.

\section{La période 2000-2017: un rapport de force État-médecins davantage déséquilibré, une imputabilité finalement imposée}

Dans un article paru en 2001, Carl-Ardy Dubois et Jean-Louis Denis distinguent trois modèles principaux d'imputabilité et les associent à trois phases différentes dans le système de santé québécois ${ }^{63}$. Le premier modèle est bureaucratique (1966-1984), où la responsabilité ultime dans le domaine de la santé incombe aux pouvoirs centraux, particulièrement au ministère de la Santé et des Services sociaux, qui a comme mandat de protéger le public et de s'assurer que les usagers ont accès à des soins de qualité. Ce modèle inscrit le processus d'imputabilité dans une ligne hiérarchique clairement déterminée et où la performance est jugée à l'aune de la conformité aux règles, à la procédure, aux codes et aux standards. Le deuxième modèle d'imputabilité est politique (1985-2000), où, désormais, la responsabilité dans le système se partage et se décentralise, au sein même du ministère de la Santé et des Services sociaux ${ }^{64}$, dans les diverses régions sociosanitaires ${ }^{65}$ et parmi les citoyens ${ }^{66}$. Plusieurs recommandations du rapport Rochon, qui se sont traduites dans la LSSS de 1991, allaient d'ailleurs dans ce sens. Enfin, le troisième modèle d'imputabilité est managérial (depuis 2000): il a pour objet non plus la protection du public ou la participation citoyenne mais principalement et surtout l'amélioration de l'efficience du système, alors que désormais seuls les résultats obtenus importent. Par ailleurs, dans ce modèle, la responsabilité entre

63. C.-A. Dubois et J.-L. Denis, préc., note 36.

64. La Loi sur l'imputabilité des sous-ministres et des dirigeants d'organismes publics, L.R.Q., c. I-4.1, adoptée en 1993 (abrogée et remplacée en 2000, comme nous le verrons ultérieurement) a rendu ces acteurs directement redevables de leur gestion à l'égard de l'Assemblée nationale du Québec.

65. Le partage et la décentralisation de la responsabilité dans le système se sont concrétisés particulièrement par la création, conformément à la LSSS de 1991, des nouvelles régies régionales, ayant une plus grande autonomie que leurs prédécesseurs, les conseils régionaux de la santé et des services sociaux.

66. Une plus grande participation citoyenne au sein des conseils d'administration des régies et des établissements ainsi que le caractère public des réunions de ces conseils d'administration sont désormais légalement prévus, ce qui confirme un rôle plus important accordé aux citoyens et à leurs représentants élus dans la gestion et le contrôle des dépenses des services de santé et de services sociaux. Voir C.-A. DuboIs et J.-L. DenIS, préc., note 36, p. 10. 
les acteurs se prescrit moins par des lois et des règlements que par des ententes contractuelles ${ }^{67}$.

Nous nous pencherons, dans la seconde partie de notre article, sur le troisième modèle d'imputabilité. Plus particulièrement, nous montrerons d'abord comment l'émergence d'une nouvelle gestion publique (NGP) ${ }^{68}$ au sein de l'administration publique québécoise a entraîné une nouvelle philosophie managériale et la mise en place d'un modèle d'imputabilité du même type au sein du système de santé (2.1). Cette trame de fond permettra ultérieurement au Parlement d'exiger que la profession médicale, dont la rémunération a, de surcroît, été substantiellement augmentée au cours des années 2000, rende des comptes au gouvernement et à la population en matière d'accessibilité aux services médicaux. Dans ce contexte, nous analyserons l'adoption du Projet de loi $\mathrm{n}^{0} 20^{69}$ en mai 2015 et la conclusion d'ententes entre le ministre de la Santé et des Services sociaux et les fédérations médicales de même que leur impact sur l'imputabilité des médecins (2.2).

\subsection{L'orientation du système de santé vers une philosophie managériale}

Sans le faire de manière exhaustive, puisque cela déborde les limites de notre texte et de nos champs d'expertise, nous trouvons néanmoins important d'énumérer certains facteurs ayant permis l'introduction de la NGP dans l'ensemble des pays industrialisés, et ce, depuis le début des années 2000. En plus de la restriction des ressources financières des années 80 , un ensemble de facteurs, comme la complexité croissante des problèmes et des attentes sociales, la fragmentation des valeurs, une détérioration des relations entre les gouvernements et les citoyens, dont rendent compte les faibles taux de participation aux élections ainsi qu'un sentiment généralisé de désenchantement à l'égard des élus, ont entraîné une remise en question de la capacité des gouvernements dans leurs formes actuelles à résoudre les problèmes économiques et sociaux auxquels ils doivent faire face et, plus globalement, une vraie crise de confiance à l'égard de l'État. Carl-Ardy Dubois souligne qu'une «conjonction d'impératifs économiques, sociaux, politiques auront poussé les gouvernements dans la grande majorité des pays de l'OCDE à engager d'ambitieux programmes de réformes

67. Sur cet aspect, voir Anne-Marie SAVARD, «Le réseau local de services de santé et de services sociaux et les ententes contractuelles entre partenaires locaux», dans Mélanie Bourassa Forcier et Anne-Marie SAVARD (dir.), Droit et politiques de la santé, Montréal, LexisNexis, 2014, p. 83.

68. L'expression est empruntée à François-Xavier Merrien, «La Nouvelle Gestion publique: un concept mythique», Lien social et Politiques, no 41, 1999, p. 95.

69. Projet de loi $\mathrm{n}^{\mathrm{o}} 20$, préc., note 2 . 
de la gestion de leurs services publics ${ }^{70} \gg$. Il résume ainsi la situation, qui décrit merveilleusement bien l'ère dans laquelle est entrée l'administration publique québécoise au tournant des années 2000: «L'imputabilité pour la performance a été placée au centre d'un discours réformiste revendiquant l'émergence d'un NMP (Nouveau Management Public) et véhiculée comme étant désormais la clé pour améliorer la qualité des services, encourager une utilisation plus efficiente des ressources, mieux adapter les services à la demande et améliorer les performances organisationnelles ${ }^{71}$.»

C'est exactement dans cette perspective qu'a été adoptée la Loi sur l'administration publique ${ }^{72}$, en 2000, qui affirme la priorité accordée par l'Administration gouvernementale, dans l'élaboration et l'application des règles d'administration publique, à la qualité des services aux citoyens et instaurant un cadre de gestion axé sur les résultats et sur le respect du principe de la transparence ${ }^{73}$. Plus particulièrement, l'article 2 de cette loi décrit le nouveau cadre de gestion gouvernemental, et nous jugeons essentiel de le reproduire intégralement tant il témoigne très clairement de la philosophie managériale adoptée par le gouvernement québécois à l'égard de l'appareil administratif pour les années à venir:

2. Le cadre de gestion gouvernementale concourt plus particulièrement:

$1^{\circ}$ à la prise en compte, dans les choix de gestion, des attentes exprimées par les citoyens en fonction des ressources disponibles;

$2^{\circ}$ à l'atteinte de résultats en fonction d'objectifs préalablement établis ;

$3^{\circ}$ à une plus grande flexibilité pour les ministères et organismes par l'adaptation des règles de gestion à leur situation;

$4^{\circ}$ à la reconnaissance du rôle des sous-ministres et des dirigeants d'organismes dans l'exercice des contrôles relatifs à la gestion axée sur les résultats;

$5^{\circ}$ à une reddition de comptes qui porte sur la performance dans l'atteinte des résultats;

$6^{\circ}$ à une utilisation optimale des ressources de l'Administration gouvernementale;

$7^{\circ}$ à l'accès, par l'Assemblée nationale, à une information pertinente sur les activités de l'Administration gouvernementale ${ }^{74}$.

70. C.-A. Dubors, préc., note 3, p. 152. Plus généralement, sur l'ensemble des aspects touchant ce que cet auteur appelle le «nouveau management public», consulter, dans le même ouvrage, les pages 148 et suivantes. Voir aussi Fr.-X. MERrien, préc., note 68.

71. C.-A. Dubols, préc., note 3, p. 152.

72. Loi sur l'administration publique, RLRQ, c. A-6.01. Notons que cette loi a abrogé la Loi sur l'imputabilité des sous-ministres et des dirigeants d'organismes publics, préc., note 64, adoptée en 1993, la nouvelle loi étant beaucoup plus large.

73. Loi sur l'administration publique, préc., note 72, art. 1. Cette loi s'applique notamment à tous les ministères du gouvernement et à plusieurs organismes publics (art. 3).

74. Id., art. 2. 
Par ailleurs, un ministère ou un organisme qui fournit directement des services aux citoyens doit rendre publique une déclaration contenant ses objectifs quant au niveau de services offerts et quant à leur qualité (déclaration de services aux citoyens $)^{75}$; chaque ministère ou organisme doit élaborer un plan stratégique devant être déposé à l'Assemblée nationale, qui comportera notamment une description de la mission du ministère ou de l'organisme en question, les orientations stratégiques, les objectifs et les axes d'intervention retenus, ainsi que les résultats visés au terme de la période englobée dans le plan $^{76}$; un ministre et le dirigeant d'une unité administrative de son ministère ou d'un organisme relevant de sa responsabilité peuvent conclure une convention de performance et d'imputabilité $^{77}$; enfin, un ministère ou un organisme doit préparer un rapport annuel de gestion (reddition de comptes) devant être déposé à l'Assemblée nationale ${ }^{78}$. Cette loi témoigne d'une perspective nouvelle selon laquelle la population n'est plus dans un rapport de confiance aveugle à l'égard du gouvernement, mais réalise au contraire qu'elle lui délègue des pouvoirs importants, notamment d'imposer des taxes, de dépenser les fonds publics ou de gérer les institutions publiques, et qu'elle est donc en droit de s'attendre, en retour, que le gouvernement en fasse un bon usage et lui rende des comptes.

Ainsi, l'État québécois a voulu insuffler un nouvel élan en matière de gestion et d'administration dans l'ensemble de la fonction publique en 2000. Dans le champ particulier de la santé, et exactement à cette époque-là, est publié le rapport d'une autre commission d'étude sur les services de santé et les services sociaux, la commission Clair $^{79}$, qui posait un diagnostic très lourd sur le système de santé et recommandait des transformations substantielles. La lecture du rapport fait ressortir en filigrane le constat selon lequel l'État est arrivé à la croisée des chemins: ou il s'ouvre sans équivoque à de nouvelles façons de faire ou il s'en va droit dans le mur, le modèle de l'État-providence ayant clairement atteint ses limites. Aux yeux de la commission Clair, les préoccupations du Québec à l'égard de son système de santé trouvent écho dans le contexte de la réflexion mise

\footnotetext{
75. Id., art. 6 et 7.

76. Id., art. 9-11.

77. Id., art. 12-23.

78. Id., art. 24-29.

79. COMMISSION D'ÉTUdE SUR LES SERVICES DE SANTÉ ET LES SERVICES SOCIAUX, Les solutions émergentes: rapport et recommandations, Québec, 2000 (ci-après « rapport Clair»). La Commission propose 95 avenues de solution (36 recommandations et 59 propositions), notamment de renforcer la première ligne, d'offrir une prestation de services globale et intégrée, de gérer plus activement l'organisation du travail et les équipes interdisciplinaires et d'y assurer une flexibilité accrue.
} 
en avant par l'Organisation mondiale de la santé (OMS) dans son document intitulé Rapport sur la santé dans le monde 2000: pour un système de santé plus performant ${ }^{80}$. Ainsi, la commission Clair s'est inspirée de ce cadre d'analyse afin de proposer une vision renouvelée du système de santé québécois pour la prochaine décennie.

Plus particulièrement, le rapport Clair s'inspire largement des principes de la NGP, dont nous avons fait état plus haut, et s'inscrit donc également dans la philosophie de la Loi sur l'administration publique. L'heure est au rationnement des ressources de l'État, à l'efficience et à l'efficacité, à l'amélioration de la performance, à la reddition de comptes et à l'imputabilité. Ce dernier terme revient d'ailleurs à un nombre incalculable de reprises tout au long du rapport et est présenté globalement comme un puissant outil permettant de développer un système de santé plus efficace et efficient. Il est approprié ici de proposer la définition que nous jugeons la plus juste et pertinente du processus d'imputabilité, parmi toutes celles que nous avons relevées. Nous retenons ainsi la définition proposée par Carl-Ardy Dubois, qui appréhende ce concept à travers quatre composantes:1) la structuration des relations entre les acteurs; 2) les mécanismes de reddition de comptes; 3) les modalités de gestion de la performance; et 4) les termes de la responsabilité des agents. Selon lui, ces quatre composantes permettent de définir le processus d'imputabilité comme «l'engagement de différents acteurs ou groupes d'acteurs impliqués dans un réseau complexe de relations dont l'enjeu est de faire en sorte que des parties assumant une responsabilité puissent rendre compte et expliquer à d'autres parties les résultats des efforts consentis et les niveaux de performance atteints ${ }^{81} »$. Nous y reviendrons.

Le rapport Clair et la philosophie managériale dont il s'inspire grandement ont entraîné des répercussions et des transformations sur le plan législatif ${ }^{82}$. En effet, bien que le cadre juridique de la Loi sur l'administration

80. ORganisation mONDiAle DE La SAntÉ (OMS), Rapport sur la santé dans le monde, 2000 - Pour un système de santé plus performant, [En ligne], [www.who.int/whr/2000/ $\mathrm{fr} /]$ (18 septembre 2017).

81. C.-A. Dubors, préc., note 3, p. 36.

82. Globalement, notons que des modifications touchant la gouverne du système de santé ont été apportées à la suite de la publication du rapport Clair. En effet, la Loi sur les agences de développement de réseaux locaux de services de santé et de services sociaux, L.Q. 2003, c. 21, a confié, en 2003, à chacune des nouvelles agences (remplaçant les régies régionales de la santé et des services sociaux) le mandat de mettre en place un nouveau modèle d'organisation, basé sur des réseaux locaux de services de santé et de services sociaux (RLS). Afin de soutenir ce nouveau modèle d'organisation et de consolider la réforme, la Loi modifiant la Loi sur les services de santé et les services sociaux et d'autres dispositions législatives, L.Q. 2005, c. 32, a été adoptée. 
publique s'applique au ministère de la Santé et des Services sociaux, le législateur a introduit en 2001 quelques dispositions au sein même de la LSSS, en imposant notamment la conclusion d'ententes de gestion et d'imputabilité ainsi que des mécanismes de reddition de comptes ${ }^{83}$. À l'époque, ces ententes de gestion et d'imputabilité devaient être conclues, d'une part, entre le ministre et chacune des régies régionales et, d'autre part, entre une régie régionale et chacun des établissements publics de sa région. Toutefois, depuis l'adoption en 2015 de la Loi modifiant l'organisation et la gouvernance du réseau de la santé et des services sociaux notamment par l'abolition des agences régionales ${ }^{84}$, où l'on a aboli le palier intermédiaire de gouvernance, soit les agences de la santé et des services sociaux (anciennes régies régionales), il est prévu que tout établissement public doit conclure directement avec le ministre une entente de gestion et d'imputabilité, contenant une définition de la mission de l'établissement, les objectifs visés pour la durée de l'entente et les principaux indicateurs permettant de rendre compte des résultats atteints ${ }^{85}$. Par ailleurs, l'établissement doit élaborer un plan d'action décrivant les moyens pris pour donner suite à l'entente et les ressources à sa disposition pour y arriver. De plus, l'établissement doit produire et déposer auprès du ministre ${ }^{86}$ un rapport annuel de

Celle-ci a introduit, entre autres, les articles 99.2 à 99.8 dans la LSSS, qui confiaient une responsabilité populationnelle à tous les prestataires de soins et de services de santé de chacun des RLS, c'est-à-dire les médecins et les pharmaciens, les organismes communautaires, les entreprises d'économie sociale et les ressources privées. Pour plus de détails sur la composition, la structure et les fonctions des RLS, voir A.-M. SAVARD, préc., note 67.

83. Loi modifiant la Loi sur les services de santé et les services sociaux et modifiant diverses dispositions législatives, L.Q. 2001, c. 24. Notons également l'adoption et l'entrée en vigueur en 2000 de la Loi sur l'équilibre budgétaire du réseau public de la santé et des services sociaux, RLRQ, c. E-12.0001, qui témoigne alors d'une volonté ferme de resserrement des dépenses publiques et qui interdit aux établissements publics de santé d'encourir un déficit à la fin d'une année financière. En ce sens, la loi aménage le processus budgétaire leur étant applicable.

84. Loi modifiant l'organisation et la gouvernance du réseau de la santé et des services sociaux notamment par l'abolition des agences régionales, RLRQ, c. O-7.2 (pour des raisons de commodité, nous ferons référence à cette loi en utilisant le numéro du projet de loi dont elle constitue l'aboutissement, c'est-à-dire le «Projet de loi $\left.n^{\circ} 10 »\right)$.

85. Id., art. 55 .

86. L'article 182.7 LSSS, préc., note 29, mentionne que le rapport annuel de l'établissement doit être transmis à l'agence mais, comme les agences ont été abolies par le Projet de loi $n^{\circ} 10$, préc., note 84, et étant donné la disposition interprétative de l'article 46, al. 2 de ce projet de loi, nous sommes d'avis que ce rapport doit dorénavant être transmis au ministre. 
gestion, pour témoigner des résultats obtenus en rapport avec les objectifs fixés dans l'entente de gestion et d'imputabilité87.

Est-ce un pur hasard que, dans ce contexte émergent, le Parlement ait voulu exiger davantage des médecins ou, en tout cas, souhaité contraindre toujours plus leur pratique médicale? En conformité avec nos développements précédents, nous répondons par la négative. Dans tous les cas, la Loi modifiant la Loi sur les services de santé et les services sociaux concernant les activités médicales, la répartition et l'engagement des médecins ${ }^{88}$ a été adoptée en décembre 2002, manifestement en vue de l'atteinte d'objectifs d'efficience et d'efficacité ainsi que dans le but de «durcir les AMP et les PREM pour mieux répartir les médecins sur le territoire ${ }^{89}{ }$. Il faut également savoir que la volonté du ministre de la Santé et des Services sociaux de l'époque, François Legault, était initialement de modifier, dans la même loi, le statut des médecins, le faisant passer d'entrepreneurs ou de travailleurs autonomes à celui de contractuels ${ }^{90}$. Or, il y a fort à parier que le climat déjà très tendu entre les médecins et le ministre, au cours de l'été 2002, à la suite de l'adoption d'une loi spéciale en juillet $2002^{91}$, aura finalement fait reculer le ministre. En effet, cette loi ordonnait notamment aux médecins désignés sur une liste de garde (conçue par le chef du département régional de médecine générale) de se présenter aux services d'urgence des établissements où ils étaient affectés et de participer aux gardes prévues sur cette liste, et ce, lorsque les services d'urgence d'un établissement étaient interrompus ou que leur maintien était menacé et

87. LSSS, préc., note 29, art. 182.7-182.10.

88. Loi modifiant la Loi sur les services de santé et les services sociaux concernant les activités médicales, la répartition et l'engagement des médecins, préc., note 54.

89. A.-C. Fourot, préc., note 5, p. 95. En plus de la redéfinition de la liste des AMP pour prioriser les services d'urgence, voir les autres changements indiqués à la note 54 . Concernant les PREM, la loi prévoit qu'ils comporteront désormais une partie sur les effectifs médicaux en «omnipratique» et une partie sur les effectifs médicaux en spécialité et que chacune de ces parties précisera, à l'égard des médecins qui travaillent en établissement, leur statut et leur volume d'activités et, à l'égard des médecins de pratique privée, leur lieu d'exercice dans la région.

90. Bien que cette volonté ne se soit pas concrétisée dans un projet de loi, le ministre s'était néanmoins publiquement exprimé ainsi sur ce sujet. François Legault avait confirmé vouloir mener «une petite révolution» dans le réseau de la santé, dans lequel les médecins deviendraient des contractuels dont la pratique de rémunération dans les hôpitaux et au sein des groupes de médecine de famille (GMF) aurait été encadrée par des contrats signés avec les établissements ou les régies régionales. Voir Robert DuTRISAC, «Legault annonce une petite révolution - Les médecins sont prêts à se battre», Le Devoir, 26 juillet 2002, [En ligne], [www.ledevoir.com/non-classe/6037/legault-annonceune-petite-revolution-les-medecins-sont-prets-a-se-battre] (18 septembre 2017).

91. Loi visant la prestation continue de services médicaux d'urgence, L.Q. 2002, c. 39. 
que le ministre estimait que cette situation nuisait ou était vraisemblablement susceptible de porter préjudice aux services médicaux auxquels toute personne a droit. De plus, cette loi ordonnait à ces médecins, lors de leur prestation de services, de ne pas diminuer ni ralentir ni modifier leur activité professionnelle de façon à interrompre ou à limiter les services médicaux. La grogne des médecins a atteint son paroxysme lorsque des régies régionales avaient utilisé cette loi pour dépêcher, par lettre d'huissier, des médecins de Montréal dans la région du Saguenay-Lac-Saint-Jean ${ }^{92}$. Pour les médecins, cette loi attaquait de plein front leur statut d'entrepreneur autonome, statut que le ministre de la Santé et des Services sociaux voyait plutôt comme "problématique puisqu'il permet aux médecins de travailler quand ils le veulent, où ils le veulent, [...] sans leur donner une véritable appartenance au réseau ${ }^{93} »$. Toujours selon le ministre, «[g]érer le système de santé avec efficacité est dans notre intérêt ${ }^{94} »$. Il est vrai que l'été 2002 aura été difficile entre les parties et qu'en conséquence cela explique certainement le recul du législateur quant à une modification du statut des médecins. Il n'en demeure pas moins que ces deux lois adoptées coup sur coup ont restreint encore davantage la pratique médicale et consacré la volonté du législateur «de voir les médecins concevoir leur pratique en fonction du système de santé au sein duquel les gestionnaires héritaient d'une autorité accrue ${ }^{95} »$.

Au cours des années suivantes, cette tendance s'est, pour ainsi dire, confirmée. En effet, dans un article analysant le pouvoir médical en rapport avec les interventions législatives au Québec de 2001 à 2008, Raymond Hudon, Rachel Mathieu et Élisabeth Martin, tout en reconnaissant que ni l'autoréglementation de la profession ni le privilège thérapeutique n'ont été remis en question pendant cette période, concluent ainsi: «En somme, il ressort de ce qui précède que le législateur a ouvertement mis en cause le statut de professionnel autonome cher aux médecins; les pressions issues d'une gestion efficace du système de santé et de services sociaux appellent

92. Isabelle PARÉ, «Legault met de l'eau dans son vin», Le Devoir, 26 septembre 2002, [En ligne], [www.ledevoir.com/index.php/non-classe/9899/legault-met-de-1-eau-dansson-vin] (19 septembre 2017).

93. Ce sont les propos du ministre de la Santé et des Services sociaux de l'époque, François Legault, cités dans Fabien DegLise, «Échec des négociations - Legault sonne la fin de la récréation», Le Devoir, 25 juillet 2002, [En ligne], [ledevoir.com/non-classe/5961/ echec-des-negociations-legault-sonne-la-fin-de-la-recreation] (19 septembre 2017).

94. Id.

95. R. Hudon, R. Mathieu et É. Martin, préc., note 5, 269 (l'italique est de nous). 
un dirigisme accentué dans la détermination des conditions d'exercice de la profession ${ }^{96}$.»

Toujours selon ces auteurs, et leurs propos rejoignent les premiers jalons que nous avons posés dans notre article, la résistance parfois féroce dont font preuve les médecins, lorsqu'il s'agit de leur autonomie professionnelle, a comme «arrière-plan la liberté quasi absolue en matière de contrôle et de régulation des soins médicaux qui avait été historiquement reconnue aux médecins jusqu'à l'adoption [...] de la Loi des hôpitaux ${ }^{97}{ }{ }$. Cependant, en raison des pressions financières ayant commencé à se faire sentir pendant les années 80 et devant une philosophie puisant aux principes d'une NGP ayant pénétré l'État québécois depuis le début des années 2000, les instances gouvernementales et législatives ne semblaient plus avoir beaucoup d'autres options que de modifier de plus en plus les modalités de la pratique médicale. Et pourtant, le contexte n'était manifestement pas encore favorable à ce changement, puisque, jusqu'à l'adoption en 2015 du Projet de loi $\mathrm{n}^{\mathrm{o}} 20^{98}$, l'État n'avait jamais mis les médecins devant un processus aussi contraignant que celui de la reddition de comptes, ce qui consacrait ainsi leur imputabilité en matière d'accès aux services de santé, comme nous le verrons dans la section 2.2.

\subsection{L'obligation (inédite) de reddition de comptes introduite par la présentation d'un projet de loi}

Dans le contexte d'un système de santé s'orientant clairement vers une philosophie managériale, qui implique notamment une plus grande imputabilité des acteurs du réseau de la santé et des services sociaux, tout comme l'ont fait plusieurs pays occidentaux d'ailleurs, divers instruments ou énoncés de position ont vu le jour pour consacrer le rôle des médecins envers la société d'aujourd'hui non plus seulement à l'égard de leurs propres patients, mais plus largement auprès de la population dans son ensemble, particulièrement en matière d'accès aux soins et aux services. C'est en tout cas dans cette perspective que le Collège des médecins du Québec a produit un rapport intitulé Nouveaux défis professionnels pour le médecin des années 2000. Commission sur l'exercice de la médecine des années

96. Id., 274. Les auteurs analysent 28 lois ayant été adoptées de 2001 à 2008 et les classent en quatre sphères d'intervention différentes: 1) les interventions touchant les conditions et l'encadrement de la pratique médicale sous l'angle d'enjeux professionnels; 2) les interventions portant sur les conditions et l'encadrement de la pratique médicale, mais sous l'angle syndical; 3) les réformes plus générales du système de santé ayant une incidence sur la pratique médicale; et 4) les lois concernant le système professionnel.

97. Id., 275.

98. Projet de loi $\mathrm{n}^{\circ} 20$, préc., note 2 . 
$2000^{99}$, dans lequel il soutient notamment ceci: «de plus en plus, la société exigera des médecins qui, tout en continuant de répondre de leurs actes médicaux accomplis à l'égard des individus, puissent répondre des soins et des services qu'ils offrent à des groupes de patients, voire à toute une population $^{100} »$.

Dans la même optique, un regroupement international d'experts américains, européens et canadiens ont publié en 2002 la Charte internationale sur le professionnalisme médical, qu'il propose comme base du contrat entre la médecine et la société101. En plus des responsabilités professionnelles traditionnelles, telles la compétence professionnelle, l'honnêteté ou la confidentialité, la Charte en précise aussi de nouvelles, par exemple l'amélioration de la qualité des soins et de leur accessibilité. Reprenant d'ailleurs les principes essentiels de cette charte internationale, l'Association des facultés de médecine du Canada a également adopté son propre texte, la Charte sur le professionnalisme médical ${ }^{102}$, où elle dispose notamment que, «[t]out en répondant aux besoins de chaque patient, les médecins sont tenus de fournir des soins de santé fondés sur la gestion prudente et rentable des moyens cliniques limités ${ }^{103}$ ». Toujours dans le même sens, le Conseil médical du Québec a publié en 2003 deux avis fouillés et détaillés, s'intitulant respectivement «Le professionnalisme et l'engagement des médecins envers la société ${ }^{104}$ » et «Imputabilité médicale et gouvernance clinique $^{105} \gg$. Enfin, à son congrès général de 2014, l'Association médicale canadienne a lancé une initiative en vue d'engager ses membres dans une importante réflexion sur le professionnalisme médical. Un des éléments déclencheurs a été le discours prononcé le gouverneur général du Canada à l'occasion de la collation des grades du Collège royal des médecins et

99. Collège Des médecins Du QuéBec, Nouveaux défis professionnels pour le médecin des années 2000, Louiseville, Service des communications du Collège des médecins du Québec, 1998.

100. Id., p. 145 et 146.

101. ABIM Foundation, ACP-ASIM Foundation et European FEdERATion of InTERnal Medicine, «Medical Professionalism in the New Millenium: A Physician Charter», Annals of Internal Medicine, vol. 136, no 3, 2002, p. 243.

102. Association des FACUlTÉS DE MÉDECINE dU CANADA, La Charte sur le professionnalisme médical, [En ligne], [afmc.ca/pdf/Charter-FRENCH.pdf] (19 septembre 2017).

103. Id., p. 2.

104. CONSEIL MÉDICAL DU QUÉBEC, Le professionnalisme et l'engagement des médecins envers la société: Avis 2003-01, 2003, [En ligne], [www.csbe.gouv.qc.ca/fileadmin/www/ Archives/ConseilMedical/Avis/2003_01_av_fr.pdf] (19 septembre 2017).

105. CONSEIL MÉDICAL DU QUÉBEC, Imputabilité médicale et gouvernance clinique. Bâtir sur la qualité et la performance des pratiques. Avis 2003-04, 2003, [En ligne], [www. csbe.gouv.qc.ca/fileadmin/www/Archives/ConseilMedical/Avis/2003_04_av_fr.pdf] (19 septembre 2017). 
chirurgiens du Canada, en $2012^{106}$. Il vaut la peine de citer ses propos, tant ils démontrent le changement de culture qui s'installe depuis quelques années au sein de la profession médicale, principalement en matière d'imputabilité et de professionnalisme médical:

Que se passera-t-il si vous ne respectez pas vos obligations aux termes du contrat social? Les Canadiens vont changer ce contrat et redéfinir le professionnalisme à votre place. On vous imposera des règlements et des modifications fort possiblement d'une manière qui diminuera ou supprimera votre privilège d'autoréglementation.

L'une des meilleures façons pour vous, et pour n'importe qui d'autre dans toute profession, d'éviter de se faire imposer des changements consiste à adopter sans relâche de nouvelles idées, à fixer et à atteindre avec ténacité des normes rigoureuses et, encore plus important, à veiller avec passion à ce que votre profession serve l'intérêt public ${ }^{107}$.

Ce discours anticipe pour le Québec ce qui s'est passé avec le Projet de loi $n^{\circ} 20$, aspect sur lequel nous reviendrons. Plus largement, ces divers instruments et réflexions marquent très certainement l'apparition d'une nouvelle culture au sein des regroupements de médecins ou de groupes qui réfléchissent à l'avenir du rôle de la profession médicale dans la société. Cependant, tout comme c'est le cas pour n'importe lequel changement de culture, la route peut être longue et parsemée de résistances, ainsi qu'en témoigne la citation suivante d'un médecin, interviewé dans le contexte des travaux de la thèse de Carl-Ardy Dubois, portant sur les transformations des systèmes de santé et les pratiques d'imputabilité: «It is evident, because of the current context of financial constraints, we have to account for resources. Having said that, it would not be acceptable, at least in the Quebec culture, that accounting for resources comes to undermine our professional autonomy ${ }^{108}$.»

Néanmoins, il est indéniable qu'à partir des années 2000 une définition renouvelée du professionnalisme médical se met en place et que les médecins, comme principaux régulateurs de l'offre et de la demande de soins, n'échapperont pas à l'application des nouveaux mécanismes de gouvernance et d'imputabilité dans le système de santé. Par ailleurs, un élément capital s'est ajouté à ce terreau, déjà fertile, et a contribué à forcer les médecins à devenir résolument imputables de leurs actes par rapport

106. AMQ, préc., note 35, p. 14. L'AMQ a aussi entamé en 2014-2015 une réflexion sur la profession médicale et la nécessité de convenir d'un nouveau contrat social. Voir Annick LePAGE, La profession médicale: vers un nouveau contrat social, Association médicale du Québec, 2015, [En ligne], [www.amq.ca/images/stories/documents/amq document_reflexion_fr_FINAL.pdf] (19 septembre 2017).

107. AMQ, préc., note 35 , p. 14.

108. C.-A. DuBoIs, préc., note 3, p. 124. 
à la population, soit les importantes hausses de rémunération qui leur ont été accordées à partir de 2007. En effet, cette année-là, sous le gouvernement Charest, des ententes entre le ministre de la Santé et des Services sociaux, Philippe Couillard, et les fédérations médicales ont été conclues relativement aux hausses de rémunération que les médecins réclamaient depuis longtemps. Comme le souligne l'Institut de recherche et d'informations socioéconomiques (IRIS), «[1]a négociation se faisait sur le principe d'un rattrapage [salarial] de la moyenne canadienne de la rémunération des médecins au plus tard en $2016^{109}$ ». Sans entrer dans les détails sur les chiffres, ce qui dépasserait notre expertise, nous voulons au moins exposer le niveau de ces hausses de rémunération. Il semble être acquis que les médecins ont obtenu, de 2007 à 2014, des augmentations annuelles moyennes de leur rémunération de 6,1 p. $100(6,4$ p. 100 pour les médecins spécialistes et 5,4 p. 100 pour les omnipraticiens) ${ }^{110}$. Selon l'IRIS, la hausse de la rémunération des médecins aura été de 82 p. 100 en dix ans (de 20102011 à 2020-2021); on ajoute que le budget de l'État consacré à ce champ de dépense dépasse celui de plusieurs ministères du gouvernement du Québec pour l'exercice financier 2015-2016 $6^{111}$.

En bref, les hausses salariales des médecins ont été très importantes au cours des dernières années mais, et c'est là que le bât blesse, ces augmentations ne semblent pas s'être traduites par un accroissement de soins et de services de santé à la population ou, en tout cas, par une amélioration de leur accessibilité. Parce qu'il semble admis que, si le gouvernement a été enclin à accorder de telles hausses salariales aux médecins, dans un contexte de ressources aussi limitées qu'actuellement, c'est en grande

109. G. HÉBERT, préc., note 31, p. 3.

110. Id., p. 5. L'IRIS ajoute que, par comparaison, pendant ce temps, les travailleurs québécois ont vu leur salaire progresser de 2,5 p. 100 en moyenne par année. Pour un état objectif de la situation liée à la rémunération des médecins concernant les ententes en vigueur de 2010 à 2015, voir aussi VÉRIFICATEUR GÉNÉRAL DU QUÉBEC, «Chapitre 2 - Rémunération des médecins: conception et suivi des ententes», dans VÉRIFICATEUR GÉNÉRAL DU QuÉBEC, Rapport du Vérificateur général du Québec à l'Assemblée nationale pour l'année 2015-2016. Vérification de l'optimisation des ressources, [En ligne], [www.vgq.gouv.qc.ca/fr/fr_publications/fr_rapport-annuel/ fr_2015-2016-VOR-Automne/fr_Rapport2015-2016-VOR-Chap02.pdf] (19 septembre 2017). Voir également l'AMQ, préc., note 35, p. 11, qui admet que l'augmentation de rémunération des médecins depuis les négociations de rattrapage de 2007 est en effet de loin supérieure à l'inflation et à la croissance moyenne des salaires de la population québécoise.

111. Voir G. HÉBERT, préc., note 31, p. 9 et 10. À titre d'exemple, l'organisme souligne que la masse salariale des médecins pour cet exercice est de 7 milliards de dollars, comparé à 4311 millions de dollars pour le ministère du Travail, de l'Emploi et de la Solidarité sociale ou à 855 millions pour le ministère de la Justice. 
partie parce qu'il considère ces gains comme des incitatifs financiers ${ }^{112}$. Or, le problème réside peut-être exactement là. En effet, il est vrai que les mesures incitatives existent depuis toujours dans le système de santé en vue d'améliorer l'accès aux soins et aux services de santé. Cependant, comme le souligne le Vérificateur général, par ces mesures, le gouvernement « vise généralement à changer le comportement des médecins en leur accordant des sommes d'argent supplémentaires s'ils remplissent les conditions préalablement définies ${ }^{113}$ ». Par exemple, on donnera un supplément pour des services rendus en horaire défavorable, un supplément selon le volume de patients inscrits ou encore une rémunération accrue du forfait de garde en disponibilité.

Or, aucune condition de cette nature n'a été négociée ni conclue dans les ententes de 2007 sur les augmentations salariales des médecins. Ainsi, est-il vraiment étonnant que l'on ne puisse établir aucune corrélation entre ces hausses de rémunération et une meilleure accessibilité aux soins et aux services de santé? Car tel est bien le cas. À la suite de ces accroissements, particulièrement de 2010 à 2014, on a plutôt observé une baisse de la productivité médicale ${ }^{114}$, ce qui a entraîné une hausse importante du coût de chacun des actes médicaux ${ }^{115}$, principalement à cause du système des enveloppes budgétaires. Pour l'IRIS, ce constat tend à «invalider les thèses de l'économie dominante qui veulent qu'un incitatif financier plus élevé s'accompagne forcément d'une hausse de l'activité encouragée ${ }^{116} \gg$. Toutefois, pour notre part, dans la mesure où les augmentations n'ont pas été négociées de manière à modifier les comportements et la pratique médicale, comme le prévoient les incitatifs financiers que nous avons mentionnés ci-dessus, ce constat n'est pas surprenant.

Il demeure vrai cependant que, dans l'esprit de la population, la conclusion selon laquelle des augmentations salariales aussi importantes accordées aux médecins, dans un contexte de restrictions des finances publiques, s'accompagnent d'une détérioration de l'accès aux soins et aux services provoque bien sûr un immense mécontentement, voire une perte de confiance de la société dans le corps médical. Comme le souligne avec justesse l'Association médicale du Québec (AMQ), aux yeux de la population, ce constat se traduit par ceci: «On paie plus cher pour moins de

112. Id., p. 8 .

113. VÉRIFICATEUR GÉNÉRAL DU QUÉBEC, préc., note 110, p. 25.

114. AMQ, préc., note 35, p. 11.

115. G. HÉBERT, préc., note 31, p. 11.

116. Id., p. 8. L'IRIS ajoute que le Québec n'est pas la seule province à avoir échoué quant à l'accroissement de l'accessibilité par l'offre d'une meilleure rémunération. 
services et on n'a pas plus accès aux services qu'avant ${ }^{117}$.» D'ailleurs, l'AMQ a commandé, en mars 2015, à la firme Léger un sondage auprès de la population au sujet de la profession médicale ${ }^{118}$. Bien que les résultats dénotent que, dans l'ensemble, la population fait encore globalement confiance à la profession médicale, de vives tensions apparaissent en rapport avec des aspects précis, notamment les séquelles des dernières négociations ayant porté essentiellement sur le rattrapage de rémunération par rapport à la moyenne canadienne, la persistance des problèmes d'accessibilité aux soins, même après la négociation d'une augmentation substantielle d'honoraires, ou encore la perception que les privilèges des médecins sont trop importants comparativement à leurs obligations ${ }^{119}$. Il ressort aussi clairement que, pour la population, «les médecins font partie des acteurs devant trouver une solution au problème d'accès aux soins ${ }^{120}$ ».

Tous ces éléments et ce contexte permettent certainement de mieux comprendre comment le Projet de loi $\mathrm{n}^{\circ} 20$ a pu être adopté en novembre 2015, parmi les réformes que le gouvernement Couillard, élu en avril 2014, souhaitait mettre en place ${ }^{121}$. Le Projet de loi $n^{\circ} 20$ constitue

117. AMQ, préc., note 35, p. 11.

118. Association médicale du Québec (AMQ), Fédération médicale étudiante du QuéBec (FMEQ) et COLLÈGE QUÉBÉCoIS DES MÉdeCINS DE FAMILle (CQMF), La profession médicale: vers un nouveau contrat social?, sondage Léger 13468-009, 2015, [En ligne], [www.amq.ca/images/stories/documents/rapport-sondageversunnouveaucontratsocial.pdf] (19 septembre 2017).

119. AMQ, préc., note 35, p. 12.

120. AMQ, FMEQ et CQMF, préc., note 118, p. 29.

121. En effet, la première réforme mise en place par le gouvernement Couillard a été de faire adopter par l'Assemblée nationale, le 6 février 2015, le Projet de loi no 10, préc., note 84. En résumé, ce projet de loi a comme objectifs principaux de favoriser et de simplifier l'accès aux services, la qualité et la sécurité des soins de même que l'efficience et l'efficacité du système de santé. Pour y parvenir, il prévoit une fusion des établissements publics de santé, passant ainsi de 182 à 29 (22 centres intégrés de santé et de services sociaux (CISSS) ou centres intégrés universitaires de santé et de services sociaux (CIUSSS) ainsi que 7 établissements non fusionnés). Ce projet de loi inclut également le passage de trois à deux paliers de gouvernance, le palier intermédiaire constitué auparavant par les agences de santé et de services sociaux disparaissant, centralisant, dans les faits, une très vaste part des pouvoirs entre les mains du ministre de la Santé et des Services sociaux. Après le Projet de loi $\mathrm{n}^{\circ} 20$, la troisième réforme que le gouvernement souhaite introduire dans le système de santé est le financement des établissements par épisodes de soins (ou financement à l'activité ou financement axé sur le patient). En bref, cette méthode de financement propose de délaisser celle des données budgétaires historiques des établissements comme référence de financement et de faire varier plutôt le financement en fonction des caractéristiques des patients, telles que les pathologies, le niveau de complexité des cas traités, la nature et le volume des épisodes de soins. La troisième réforme n'est pas encore à l'ordre du jour législatif pour l'instant, mais elle devrait l'être sous peu. 
vraisemblablement l'aboutissement ultime mais aussi inévitable de cette longue évolution de la pratique médicale depuis l'instauration du système public de santé au Québec en 1970. Tant sur la forme que sur le fond, ce projet de loi est inédit. Voyons d'abord ce qu'il en est sur la forme. Traditionnellement, à la suite de la présentation de projets de loi qui touchent de manière importante à la pratique médicale ou à l'autonomie professionnelle, les regroupements de médecins utilisent divers moyens, notamment des sorties dans les médias, des menaces de grèves ou des manifestations publiques ${ }^{122}$, réussissent la plupart du temps à faire reculer le gouvernement sur certains aspects et finissent par obtenir un «produit fini» leur convenant, au moins partiellement ${ }^{123}$. La loi est bien souvent le résultat d'un compromis entre les parties, comme nous l'avons vu, et le gouvernement obtient des résultats parfois différents de ceux qu'il espérait au départ. Dans le contexte du Projet de loi $n^{\circ} 20$, les choses se sont passées différemment. En effet, il a été présenté le 28 novembre 2014. Dès le 13 décembre 2014, le conseil général de la Fédération des médecins omnipraticiens du Québec (FMOQ) adopte une résolution en vue de déterminer d'autres moyens d'atteindre l'objectif qui consiste à améliorer l'accès aux services de médecine de famille. C'est le début d'une période au cours de laquelle les fédérations médicales tenteront de faire reculer le gouvernement non pas sur les objectifs à atteindre, ou sur les résultats, mais plutôt sur les moyens pour y parvenir. Dans ce cadre, l'élément différent est aussi que, certes, les instances exécutives des fédérations médicales ont fait quelques sorties dans les médias, mais aucun moyen de pression ni menace de grève n'ont été utilisés. Finalement, les négociations menées entre les fédérations médicales et le ministère de la Santé et des Services sociaux ont abouti à deux ententes signées, l'une avec la FMOQ, le 2 juin 2015, et l'autre avec la FMSQ, le 11 janvier 2016, ce qui a permis de surseoir, temporairement, à l'application de nombreuses dispositions législatives si, de manière permanente, au 31 décembre 2017, les résultats négociés sont au rendezvous ${ }^{124}$. Nous reviendrons plus bas sur le contenu de ces ententes. Cette fois, en raison de la conjoncture actuelle, représentant l'aboutissement d'une longue évolution que nous avons relatée, le gouvernement se trouvait

122. Il va sans dire que les regroupements de médecins connaissent les vœux du gouvernement avant même la présentation de projets de loi les concernant. Ces échanges souvent informels se font selon des règles ou des us et coutumes appartenant aux acteurs visés et dont il est difficile de connaître officiellement les modalités. Cela n'est de toute manière pas essentiel aux fins de notre article.

123. Nous en voulons pour preuve, notamment, ce que nous avons mis en lumière antérieurement concernant l'adoption de la seconde version de la LSSS, en 1991.

124. En effet, bien que le Projet de loi n ${ }^{\circ} 20$ soit entré en vigueur le 10 novembre 2015, de nombreuses dispositions législatives ne le sont toujours pas. 
clairement en position dominante lors des négociations. Il n'aura pas eu à reculer sur les résultats souhaités, mais seulement sur les moyens pour y arriver, moyens qu'il préférait peut-être d'ailleurs, tout en laissant planer le spectre de la loi, comme une épée de Damoclès, à défaut de résultats livrés par les médecins. L'AMQ reconnaissait d'ailleurs que, avec le Projet de loi $\mathrm{n}^{\mathrm{o}} 20$, l'État et son ministre avaient tenté de changer les règles du jeu des négociations entre les fédérations médicales et le gouvernement, et que ce projet de loi avait cristallisé le rapport de force entre les parties, à l'avantage, cette fois, du gouvernement et de son ministre de la Santé et des Services sociaux ${ }^{125}$.

Nous verrons maintenant ce qu'il en est du Projet de loi $n^{\circ} 20$ sur le fond. Celui-ci a pour objet d'optimiser l'utilisation des ressources médicales et financières du système de santé afin d'améliorer l'accès aux services de médecine de famille et de médecine spécialisée. À cette fin, il prévoit certaines obligations applicables à la pratique des médecins qui participent au régime d'assurance maladie du Québec. Ainsi, il impose notamment aux médecins de famille participants au régime public, dans la mesure prévue par règlement du gouvernement, de satisfaire aux obligations suivantes: assurer, individuellement ou avec d'autres médecins au sein d'un groupe de médecine de famille (GMF), le suivi médical d'une clientèle constituée d'un nombre minimal de patients ${ }^{126}$ et exercer, auprès des usagers d'un établissement, un nombre minimal d'heures d'activités médicales autorisé par le département régional de médecine générale de la région visée ${ }^{127}$. Il est également prescrit que les médecins de famille doivent se rendre disponibles auprès des personnes assurées en utilisant un système de prise de rendez-vous mis en place conformément au Projet de loi $\mathrm{n}^{\mathrm{o}} 20^{128}$. À l'égard des médecins spécialistes, celui-ci prévoit qu'ils doivent notamment, dans le contexte du mécanisme d'accès priorisé aux services spécialisés,

125. AMQ, préc., note 35, p. 11.

126. Projet de loi $\mathrm{n}^{\circ} 20$, préc., note 2 , art. 4 , al. 1 (1).

127. Id., art. 4, al. 1 (2). Notons que, pour ces deux obligations, le règlement peut notamment prévoir:

- l'âge à compter duquel un médecin peut y être soustrait;

- les modalités de suivi de la clientèle;

- le nombre minimal de patients devant être suivis;

- les activités médicales pouvant faire l'objet d'une autorisation;

- le nombre minimal d'heures d'activités médicales devant être exercées;

- les règles particulières applicables lorsqu'un médecin souhaite exercer des activités médicales dans plus d'une région;

- toute autre condition qu'un médecin doit respecter afin de satisfaire à ces obligations (art. 4, al. 2).

128. Id., art. 11. 
recevoir en consultation, ailleurs qu'au service d'urgence d'un établissement, des patients qui ne sont pas des usagers admis dans un centre exploité par un établissement ${ }^{129}$. Les médecins spécialistes exerçant en centre hospitalier doivent aussi assurer en tant que médecins traitants, avec les autres médecins de la même spécialité, la prise en charge et le suivi médical d'usagers admis dans ce centre ${ }^{130}$. Voilà brièvement résumées les principales obligations qu'impose le Projet de loi $\mathrm{n}^{\mathrm{O}} 20$ aux médecins omnipraticiens et spécialistes qui participent au régime public de santé. Par ailleurs, à défaut pour un médecin de se conformer à ces obligations, le gouvernement a prévu la réduction de sa rémunération par la Régie de l'assurance maladie du Québec (RAMQ) ${ }^{131}$.

Évidemment, les mémoires des fédérations médicales déposés à la Commission de la santé et des services sociaux de l'Assemblée nationale sur le Projet de loi $\mathrm{n}^{\circ} 20$ ont été clairs et unanimes sur son caractère totalement inacceptable. Pour la FMOQ, «le cumul de toutes les nouvelles obligations qui devront être effectuées sous peine de sanctions, à la fois en établissement et hors établissement, enfermera totalement les médecins dans un cadre à ce point rigide, qu'il brisera toute autonomie professionnelle $^{132}$ ». Quant à la FMSQ, s'insurgeant contre le fait que le Projet de loi $\mathrm{n}^{\circ} 20$ a été déposé alors que des rencontres sur l'accessibilité aux soins et services avaient été amorcées quelques semaines auparavant entre les fonctionnaires du Ministère et ses propres représentants, elle considère que ce projet de loi «renie le bien-fondé de la négociation et le partenariat avec les médecins spécialistes et adopte une approche coercitive en mettant en place des obligations unilatérales, des quotas et des pénalités ${ }^{133} »$. De son côté, l'AMQ estime que la modification de la prise en charge des patients induite par le Projet de loi ${ }^{\circ} 20$ est plus qu'une simple question de nombre d'individus: «elle vise à réguler la pratique médicale, à revoir l'offre de services des médecins et, finalement, à transformer l'exercice de la

129. Id., art. 13 .

130. Id., art. 14.

131. Voir le Projet de loi n ${ }^{\circ} 20$, préc., note 2, art. 26. De plus, celui-ci donne des pouvoirs de surveillance aux présidents-directeurs généraux d'établissements, aux départements régionaux de médecine générale ainsi qu’à la RAMQ. Voir les articles 23, 24 et 25.

132. FÉdÉRATION DES MÉdECINS OMNIPRATICIENS DU QUÉBEC (FMOQ), Mémoire de la FMOQ présenté à la Commission de la santé et des services sociaux concernant le projet de loi $n^{\circ}$ 20, p. 8, [En ligne], [fmoq.s3.amazonaws.com/affaires-syndicales/memoires/ PL20-Memoire-FMOQ-19mars2015.pdf] (19 septembre 2017).

133. FÉdÉRATION DES MÉDECINS SPÉCIALISTES DU QUÉBEC (FMSQ), Mémoire de la Fédération des médecins spécialistes du Québec. Projet de loi no 20. Déposé à la Commission de la santé et des services sociaux, p. 5, [En ligne], [www.fmsq.org/documents/10275/13957/ memoire17032015.pdf] (19 septembre 2017). 
médecine ${ }^{134} \gg$. Toutefois, l'AMQ ajoute ces propos, qui nous paraissent bien représenter la réalité actuelle concernant la régulation de la pratique médicale et qui méritent d'être reproduits:

Inspirés des pratiques issues du domaine privé, les réformistes tentent de mesurer, de standardiser et d'optimiser la performance de la pratique médicale. Pour ce faire, ils repensent et renouvellent les mécanismes de régulation; les modèles traditionnels d'imputabilité semblent aujourd'hui ne plus suffire à garantir l'excellence de la pratique médicale. En somme, le contrôle exercé par les pairs, contrôle qui était auparavant légitimé par les connaissances, les standards de pratiques, les valeurs morales et la réputation, n'est plus considéré comme valable pour témoigner de l'efficience des membres de la communauté médicale. En raison d'un désinvestissement de ses membres au niveau de la gouvernance clinique, la communauté médicale pourrait assister à la diminution de son autonomie clinique et de sa capacité à s'autoréguler ${ }^{135}$.

Comme nous l'avons mentionné ci-dessus, et évidement après de nombreux échanges et pourparlers entre les parties, les deux fédérations médicales et le ministre de la Santé et des Services sociaux ont finalement signé des ententes: elles ont eu pour effet de suspendre l'application des dispositions du Projet de loi $\mathrm{n}^{\mathrm{o}} 20$ imposant les nouvelles obligations aux médecins, dans un premier temps jusqu'au 31 décembre 2017. En résumé, nous pouvons affirmer que les ententes substituent aux obligations «individuelles » de rendement, assorties de sanctions financières en cas de défaut, des obligations «collectives» de rendement, accompagnées de cibles fixées en fonction d'un échéancier précis, au terme duquel elles devront avoir été atteintes, faute de quoi les dispositions du Projet de loi $\mathrm{n}^{\mathrm{o}} 20$ entreront en vigueur. Si toutefois les cibles sont atteintes, les ententes prévoient plutôt qu'un nouveau projet de loi sera adopté avant la fin de la session parlementaire du printemps 2018, ou au plus tard le 30 juin 2018, lequel abrogera les dispositions en question.

Plus précisément, pour l'ensemble des médecins de famille, l'entente prévoit notamment une cible d'inscription et de suivi de patients ( 85 p. 100 de la population du Québec) et une cible d'assiduité (80 p. 100), cibles qui devront être atteintes au plus tard au 31 décembre 2017, ainsi que des indicateurs de performance en vue d'évaluer conjointement l'atteinte de ces cibles ${ }^{136}$. Quant aux médecins spécialistes, l'entente prévoit les quatre éléments d'accessibilité suivants:

134. A. LePaGe, préc., note 106, p. 18.

135. Id., p. 23.

136. Selon le site Web de la FMOQ, en date du 8 février 2017, le taux d'inscription était d'environ 75 p. 100, tandis que le taux d'assiduité approche 80 p. 100, ce qui correspond à la cible. Voir FÉdÉRATION DES MÉDECINS OMNIPRATICIENS DU QUÉBEC (FMOQ), «Accès aux médecins de famille: une prise en charge inédite l'automne dernier», 
1) l'amélioration de l'accès aux consultations spécialisées selon des délais associés à la priorité clinique attribuée à un patient ;

2) la diminution des délais associés aux consultations spécialisées demandées au service d'urgence d'un établissement;

3) l'augmentation du taux de prise en charge de patients hospitalisés par des médecins spécialistes en tant que médecins traitants; et

4) la priorisation des interventions chirurgicales de patients en attente depuis plus d'un an.

Pour chacun de ces éléments, l'entente prévoit des indicateurs et des cibles, à atteindre d'ici le 31 décembre 2017.

Évidemment, les ententes contiennent de nombreuses autres dispositions qu'il nous est malheureusement impossible d'exposer et d'analyser ici. Nous tenons plutôt à faire ressortir les constats notables qui se dégagent du processus et des résultats inédits qui entourent la réforme du gouvernement Couillard sur l'accès aux services de médecine de famille et de médecine spécialisée. Force nous est de conclure que, bien que les moyens diffèrent, tant le Projet de loi $\mathrm{n}^{\circ} 20$ que les ententes qui s'en sont finalement suivies contiennent, pour la première fois, des objectifs clairs d'imputabilité imposés aux médecins en matière d'accès. Il ne s'agit plus simplement de contraindre ou d'orienter la pratique médicale selon des modalités fixées dans une loi, mais bien d'exiger des médecins qu'ils fournissent des résultats et qu'ils rendent des comptes, que ce soit individuellement ou collectivement. En effet, si nous reprenons les termes de la définition du concept d'imputabilité proposée par Carl-Ardy Dubois, que nous avons indiquée plus haut ${ }^{137}$, il se dégage clairement que, pour la première fois depuis l'instauration du régime public de santé, un véritable lien d'imputabilité est créé entre les médecins et le gouvernement et, ultimement, la population. Ce n'était certainement qu'une question de temps, mais cela devait se produire. Certains ont fait grand bruit autour du «style» et de la forte personnalité du ministre de la Santé et des Services sociaux actuel pour expliquer qu'il a été possible de parvenir à imposer de telles obligations de rendement aux médecins. Cependant, notre article démontre plutôt qu'une succession d'évènements et d'éléments se sont mis en place depuis les débuts du régime public d'assurance maladie au Québec pour faire en sorte que, ultimement,

communiqué de presse, 8 février 2017, [En ligne], [www.fmoq.org/affaires-syndicales/ communications/communiques/une-prise-en-charge-inedite-1-automne-dernier/] (19 septembre 2017).

137. Voir infra, section 2.1. 
les médecins aussi deviennent imputables en matière d'accès aux soins et aux services de santé.

\section{Conclusion}

Dans son ouvrage intitulé De la démocratie en Amérique, Alexis de Tocqueville soutient ceci: «Les peuples se ressentent toujours de leur origine. Les circonstances qui ont accompagné leur naissance et servi à leur développement influent sur tout le reste de leur carrière ${ }^{138}$.» Pourraiton transposer ces propos à la mise en place du système public de santé, qui s'est ressenti pendant très longtemps des importantes concessions alors faites aux médecins, laissant intouchables leur statut de travailleurs autonomes et le mode de rémunération? Notre analyse historique de l'encadrement normatif de la pratique médicale au Québec nous pousse à répondre par l'affirmative. En effet, si l'État s'est buté, pendant de très nombreuses années, à une forte résistance médicale, c'est bien parce que les compromis faits au début des années 70 ont marqué les rapports de force entre l'État et les médecins, à l'avantage de ces derniers. Cependant, plusieurs éléments se sont conjugués au fil du temps pour finalement instaurer un nouvel équilibre entre les parties, cette fois en faveur de l'appareil étatique.

L'objectif de notre article était donc de relater l'évolution et d'analyser les facteurs ayant permis ce passage de l'autonomie professionnelle des médecins à une imputabilité dans le système de santé, particulièrement en matière d'accès aux soins et aux services. Comme nous l'avons vu dans la première partie, bien que la pratique de la médecine ait pris un tournant majeur avec la mise en place d'un système public de santé au début des années 70, les médecins auront néanmoins réussi à conserver une marge de manœuvre appréciable dans la pratique de leur profession. Cependant, quelques années plus tard, les difficultés financières de l'État québécois l'ont poussé à contraindre davantage l'action et la pratique des médecins. Il n'en demeure pas moins que les résistances et le pouvoir de la profession médicale au cours des 30 premières années du système permettaient encore la préservation d'une large part d'autonomie professionnelle. Dans la seconde partie de notre texte, nous nous sommes intéressée à l'émergence d'une NGP au sein de l'administration publique québécoise et à ses conséquences sur le système de santé, principalement l'introduction d'une philosophie managériale. Misant notamment sur la gestion par les résultats, la transparence et la reddition de comptes, cette nouvelle orientation a incontestablement rendu possible l'adoption du Projet de loi $\mathrm{n}^{\mathrm{o}} 20$ ainsi que la conclusion d'ententes entre le ministre de la Santé et

138. Alexis De Tocqueville, De la démocratie en Amérique, t. 1, chap. 2. 
des Services sociaux et les fédérations médicales, ententes qui consacrent désormais une imputabilité des médecins à l'égard du gouvernement en matière d'accès aux soins et aux services de santé.

Cela étant dit et pour conclure, la manière d'imposer cette imputabilité était-elle la meilleure? En effet, la forme d'imputabilité présente dans le Projet de loi $\mathrm{n}^{\mathrm{o}} 20$ et même dans les ententes est axée sur le contrôle formel des médecins par le gouvernement. Selon Carl-Ardy Dubois, cette forme part du principe selon lequel la poursuite de l'intérêt personnel est le moteur principal du comportement humain et que, donc, la mobilisation des individus autour d'objectifs collectifs ou publics n'est possible qu'en contraignant les comportements par un ensemble de mécanismes formels ${ }^{139}$. Tout porte à croire que c'est exactement le présupposé du gouvernement et de son ministre de la Santé et des Services sociaux, dans le contexte actuel. Or, cette façon de procéder pourrait devenir contreproductive et receler des conséquences négatives, que nous ne saurions passer sous silence. Voici ce que Carl-Ardy Dubois écrivait dans sa thèse en 2002, alors que le Projet de loi $n^{\circ} 20$ n'existait pas: «Une culture de blâme peut aller à l'encontre de la prise d'initiative. Des règles trop rigides peuvent entraver l'autonomie des professionnels et les empêcher d'exercer adéquatement leur jugement. Des systèmes de gestion axés sur le contrôle de la conformité peuvent détacher l'attention de l'atteinte des résultats. L'accent sur les sanctions et le refus du droit à l'erreur s'accommode (sic) mal de l'innovation ${ }^{140}$.»

Carl-Ardy Dubois, pour sa part, prône des formes plus socialisantes d'imputabilité, reconnaissant les individus davantage comme des acteurs sociaux susceptibles d'avoir un comportement honnête et coopératif et pouvant, de manière spontanée et délibérée, être motivés à contribuer au bien commun ${ }^{141}$. Bien que les analyses de cet auteur relèvent davantage du champ de l'administration de la santé que du droit, ses propos nous semblent toutefois suffisamment pertinents pour être au moins soulevés, en terminant notre article. L'avenir nous dira si le pari et une telle imposition d'imputabilité aux médecins par le ministre de la Santé et des Services sociaux et le gouvernement auront su porter des fruits.

139. C.-A. Dubois, préc., note 3, p. 93 et 94.

140. Id., p. 94.

141. Id., p. 96. 